\title{
On Applying the Critical Thermal Maxima Method to Investigate Ecologically-Relevant Questions in Wild Fishes
}

\author{
By \\ Jessica Emilie Desforges
}

B.Sc. with Honours in Applied Animal Biology, University of British Columbia, 2020
A thesis submitted to the Faculty of Graduate Studies and Research
In partial fulfillment of the requirements for the degree of
Masters of Science
in
Biology
Carleton University,
Ottawa, Ontario

(C)2021, Jessica Emilie Desforges 


\section{Dedication}

To the amazing fish and fishy-folks that I was fortunate enough to meet during the completion of this thesis, including my insightful supervisor Dr. Steven Cooke, who acted as a great mentor along the way. To Dr. Kim Birnie-Gauvin, who not only acted as a mentor, but who also had the chance to pull her hair out with me while struggling with a series of unanticipated events in Denmark - from hail storms to flooded waders, frozen feetses, bad jokes, and persistent bad luck. Thank you for all the laughs, discussions, dedication, and above all, putting up with all my crazy ideas over the past two years. To the other co-authors I had a chance to collaborate with - I have learned so much from every single one of you. The words of encouragement, insightful comments, and discussions we have had along the way have inspired me to pursue further work in this field. Finally, thank you to my friends, family, and partner in crime for being the source of my motivation and encouraging me even on my worse days. You have all played an incredibly valuable role in the completion of this thesis. 


\begin{abstract}
The role of temperature in mediating the behaviour and physiology of fishes is becoming more apparent, as climate change exacerbates the frequency and severity of extreme weather events. Among the many methods used to estimate upper thermal limits, the Critical Thermal Maxima method has been the dominant approach partially due to its relative simplicity in experimental design. However, several concerns have been raised about the ecological relevance of this method, particularly due to the use of rapid rates of thermal ramping. In this thesis, I begin by reviewing the ecological relevance of CTmax. I discuss methodological concerns and limitations, while outlining opportunities to address these concerns and apply CTmax in an ecologically-relevant way. I then provide an example of a field-based study that evaluates the role of CTmax estimates in accounting for variation in life-history traits and fitness in a semianadromous population of juvenile brown trout (Salmo trutta).
\end{abstract}




\section{Acknowledgements}

I would like to thank my supervisor, Dr. Steven Cooke, for his support and confidence in my abilities. I would also like to thank Dr. Kim Birnie-Gauvin, Dr. Kim Aarestrup, and Michael Skafte for their efforts in assisting with the logistics, field work, and experimental design involved in this international field-based project. Thank you to all my other co-authors; Amanda Bates, Fredrik Jutfelt, David McKenzie, Michael Lawrence, Erika Eliason, and Kathleen Gilmour. I would also like to thank Ben Hlina, Chris Elvidge and Alexandre Gagnon for their assistance with data analysis and for providing comments. Support for my research was provided by the Natural Sciences and Engineering Research Council (NSERC) Strategic and Discovery Grants to S.J.C., who is also supported by the Canada Research Chairs program. This project was also partly funded through the Danish Rod and Net Fish License Funds. 


\section{Co-Authorship}

Chapter 2: On the ecological relevance of critical thermal maxima in fish. Jessica Emilie Desforges, Kim Birnie-Gauvin, Amanda E. Bates, Fredrik Jutfelt, Erika J. Eliason, David McKenzie, Michael Lawrence, Kathleen Gilmour, and Steven J. Cooke.

While this study is my own, the research was undertaken as part of a collaborative effort and each co-author played a valuable role in its completion. The project was conceived by Desforges and Cooke. All writing was conducted by Desforges. Birnie-Gauvin, Bates, Jutfelt, Eliason, McKenzie, Lawrence, and Cooke provided insight, comments, and feedback on the manuscript.

\section{Chapter 3: Upper thermal tolerance indicated by CTmax fails to predict migration} strategy and timing, growth, and predation vulnerability in juvenile brown trout. Jessica Emilie Desforges, Kim Birnie-Gauvin, Kim Aarestrup, and Steven J. Cooke.

While this study is my own, the research was undertaken as part of a collaborative effort and each co-author played a valuable role in its completion. The project was conceived by Desforges and Cooke. All writing was conducted by Desforges. Desforges, Birnie-Gauvin, and Aarestrup assisted with experimental design and data collection. Desforges, Birnie-Gauvin, Aarestrup, and Cooke provided insight, comments, and feedback on the manuscript. 


\section{Table of Contents}

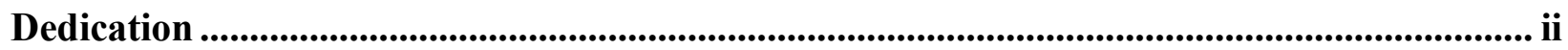

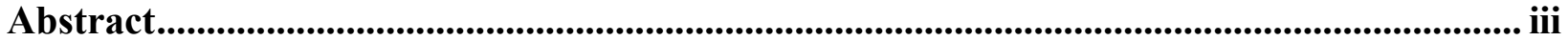

Acknowledgments ............................................................................................................................... iv

Co-Authorship .......................................................................................................................................

List of Tables ................................................................................................................. vii

List of Figures................................................................................................................................ ix

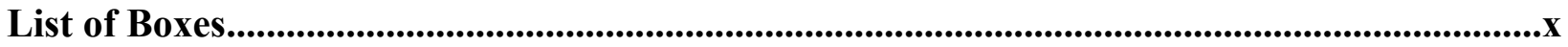

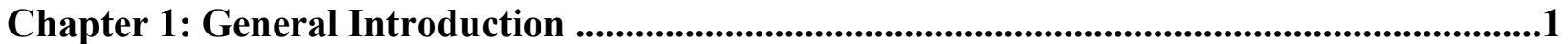

Chapter 2: On the Ecological Relevance of Critical Thermal Maxima Measurements for

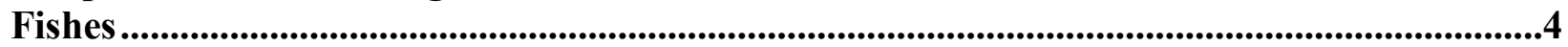

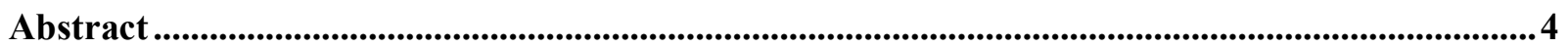

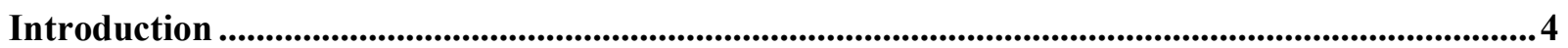

The history of CTmax: Past applications and limitations ..........................................................................

Questioning the ecological relevance of CTmax as a measure of upper thermal tolerance............11

Why is the rate of thermal ramping so important when designing CTmax experiments? ................................11

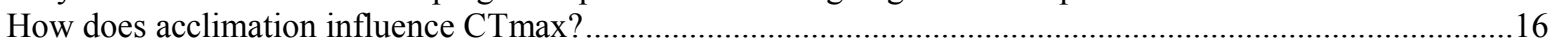

How does CTmax compare to other estimates of thermal tolerance to determine thermal performance? ..........20

What are thermal safety margins and how can CTmax be used to determine them? ....................................25

Is CTmax repeatable, and what does that tell us about heritability and adaptive potential?..........................29

Conclusion ..........................................................................................................................................

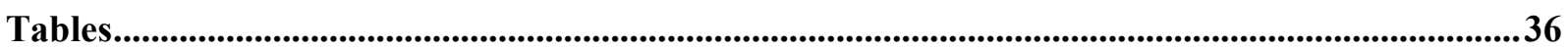

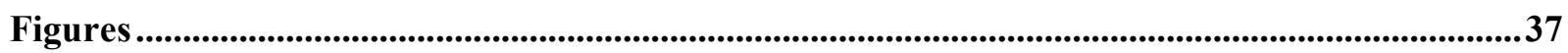

Chapter 3: Upper thermal tolerance indicated by CTmax fails to predict migration strategy and timing, growth, and predation vulnerability in juvenile brown trout (Salmo trutta).....41

Abstract .........................................................................................................................................................4

Introduction .........................................................................................................................................42

Materials and Methods .............................................................................................................................4

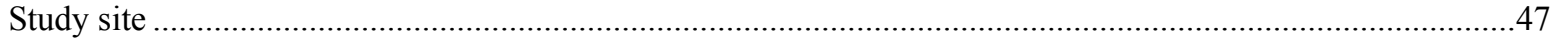

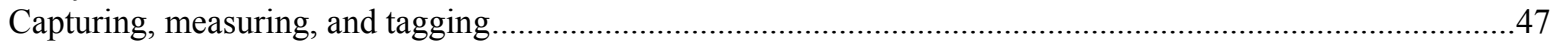

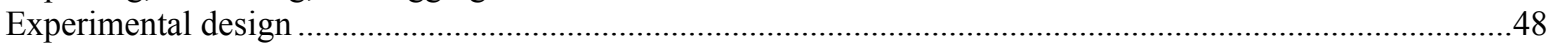

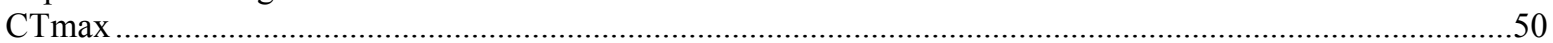

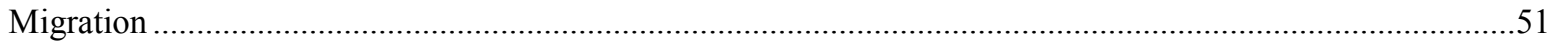

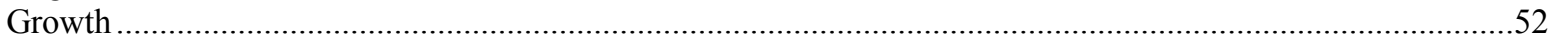

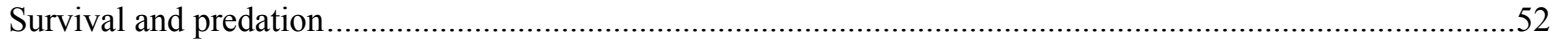

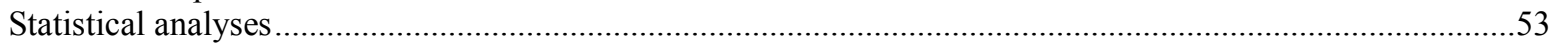

Results.........................................................................................................................................55

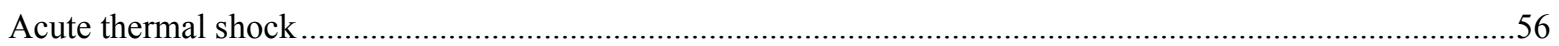

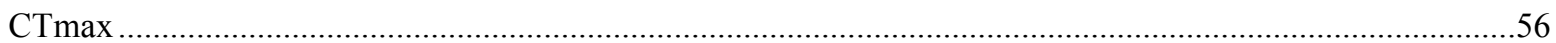

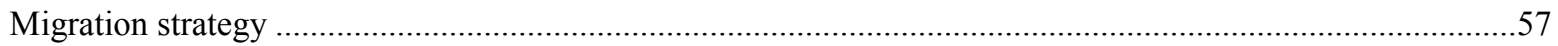

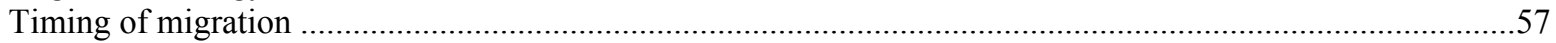


Growth.

Predation

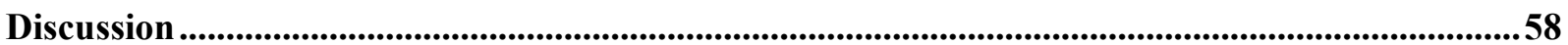

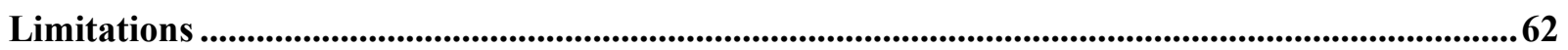

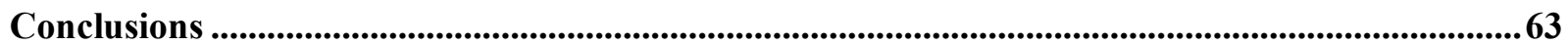

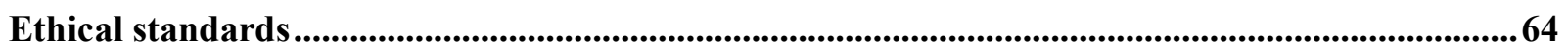

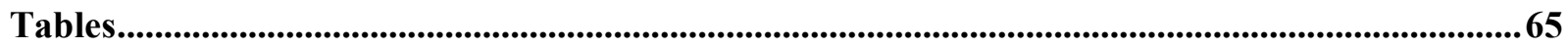

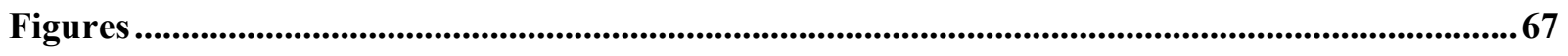

Chapter 4: General Discussion and Conclusion............................................................70

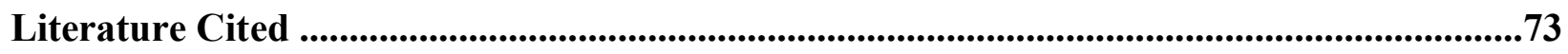




\section{List of Tables}

Table 2-1. Summary of considerations for making CTmax more ecologically relevant .36

Table 3-1. Summary of tagged fish. Mean initial length $(\mathrm{cm})$, mass $(\mathrm{g})$, and condition $(\mathrm{K})$ for individually tagged Salmo trutta for each treatment group ( \pm s.d.). The proportion of fish either migrating, residing or of unknown strategy within each group is shown as a percentage. Subscript letters indicate significant differences between treatments. Note that predated fish include residents, migrants, and unknown fish

Table 3-2. CTmax by final status. Total number $(\mathrm{N})$, mean \pm s.d., minimum, and maximum cumulative temperature (cumulative degree minutes) and temperature $\left({ }^{\circ} \mathrm{C}\right)$ at loss of equilibrium for individually tagged Salmo trutta exposed to thermal ramping based on status as of June $8^{\text {th }}$, 2020. Note that Predation category includes fish categorized as migrants, residents, and unknown

Table 3-3. Final statuses metric summary. Total number $(\mathrm{N})$, mean \pm s.d., and range of body metrics obtained during initial sampling of control and CTmax Salmo trutta in February 2020 according to final statuses. Note that Predation category includes fish categorized as migrants, residents, and unknown 


\section{List of Figures}

Figure 2-1. Thermal Performance Curve. Conceptual diagram of thermal performance curves (TPC), with critical thermal minima $\left(\mathrm{CT}_{\min }\right)$ and critical thermal maxima $\left(\mathrm{CT}_{\max }\right)$ represented where performance approaches zero, the thermal optima ( $\left.\mathrm{T}_{\mathrm{opt}}\right)$ represented at a temperature where the organism exhibits maximal performance, and thermal safety margins (TSM), denoted by the range of temperatures between $\mathrm{T}_{\text {opt }}$ and $\mathrm{CT}_{\max }$. Performance indices vary, but typically include metabolic rate, aerobic scope, swimming speed, or growth depending on research interests

Figure 2-2. CTmax flowchart. A conceptual diagram outlining the links between methodological inputs, research interests, and potential outcomes

Figure 3-1. Gudsø study site map. Location of study site, Gudsø stream, Jutland, Denmark. PIT stations are indicated by black circle. Traced lines represent areas where the stream runs and the outflow location of the stream into the Kolding Fjord

Figure 3-2. CTmax and mass/condition. Modelled Cumulative Degree Minutes (CDM) as a function of (A) mass (log-log transformed, with initial condition held at mean values) and (B) initial condition factor (K, with mass held at mean values) in juvenile Salmo trutta. (A) suggests a positive relationship between mass and CDM while $(\mathbf{B})$ suggests a negative relationship between condition factor and CDM. Shaded areas represent $95 \%$ confidence intervals

Figure 3-3. Migration timing. (A) Modelled probability of timing of migration (days spent in river after sampling) as a function of mass (log-log transformed, with initial condition held at mean values) in juvenile Salmo trutta illustrating a significant negative relationship between mass and migration timing $(Z=-2.685, P=0.007)$. Shaded areas represent $95 \%$ confidence intervals. (B) Distribution of initial mass $(\mathrm{g})$ demonstrating significantly greater mass $(P=$ $0.008)$ of early migrants ( 0 -30 days post-sampling, $N=54)$ compared to late migrants $(31+$ days post-sampling, $N=88$ ) 


\section{List of Boxes}

Box 2-1. OCLTT. On the use of the oxygen-capacity limited thermal tolerance hypothesis to investigate performance in fishes....

Box 2-2. Research needs. We identified research priorities by using word cloud software to extract the top 50 words from each of the five considerations discussed in the previous sections. We then generated a subsequent word cloud using these extracted words to narrow down the ten most common terms discussed. This method yielded the following list of words: climate, estimates, stress, conditions, change, acute, rate, time, species, and fitness. We opted to add the term context, as it is central to research in the field of thermal biology. As such, we present the following list of nine themes (acute, rate, and time were grouped) to help focus research aimed at optimizing the use of CTmax in the context of ecology ...............................................................40 


\section{Chapter 1: Introduction}

The field of thermal biology has been receiving increasingly more interest over recent years as researchers attempt to identify and protect vulnerable populations or make predictions about individual, population, community, or ecosystem-level responses to climate change induced thermal stress. Fish, which are ectothermic animals, have been a particular focus given their reliance on external environmental conditions to regulate body temperature. While several studies forecast a greater frequency of heatwaves and droughts (IPCC 2014, Seneviratne et al. 2014, IPCC 2021), ectotherms have been found to already be coping with high and more variable temperatures (Morgan et al. 2019). In fact, there have already been documented cases of mass mortality events in fishes, where droughts and high temperatures exceeded critical thermal limits (e.g., Wegner et al. 2008; Finnegan et al. 2012; Penn et al. 2018; Vertessy et al. 2019; Genin et al. 2020). As such, there currently exists a race between climate change and expanding our current knowledge and tools required to mitigate thermal stress in ectotherms. Even in the absence of climate change, human activities such as nuclear power generation or industrial applications can lead to rapid changes in water temperature (Coutant and Brook 1970).

Given the importance of temperature in modulating the distribution, behaviour, and physiology of wild fish, accurately describing thermal limits has been particularly important in the field of fish biology. Thermal tolerance generally refers to a specific thermal window that is bound by critical limits within which an individual's fitness is greater than zero (MacMillan 2019). Simply stated, thermal tolerance is a range of temperatures in which an individual can meet basic physiological demands. Although thermal tolerance can be measured in a variety of ways, the boundaries are frequently characterized by impaired performance, loss of equilibrium, or death (Becker and Genoway 1979; Lutterschmidt and Hutchison 1997). Among these 
methods, the critical thermal maxima (CTmax) method was first described in 1944 by Cowles and Bogert, and has evolved to become the dominant protocol for estimating thermal limits in ectotherms.

Briefly, the CTmax method describes acute upper thermal limits that are marked by loss of equilibrium (LOE). In general, fish are acclimated to a specific temperature, after which they are subjected to a steady rate of thermal ramping that ranges from minutes to hours. Although little is known about the underlying mechanisms of LOE (e.g., Wang et al. 2014, Ern et al. 2016, 2017, Jutfelt et al. 2019), researchers have broadened the application of CTmax in fish ecology. In attempt to investigate a range of research questions, fundamental elements to the CTmax protocol, such as the acute rate of ramping or acclimation, have been altered, ignored, and often criticized. Many have argued that the acute rates of thermal ramping required for CTmax experiments are not realistic (e.g., Mora and Maya 2006, Vinagre et al. 2015), or that alternative endpoints are ultimately better descriptors of upper thermal tolerance (e.g. Blasco et al. 2021). As a result, the literature includes many different approaches to measuring CTmax, which often yield thermal limit estimates that are not comparable across studies.

In this thesis, I focus on the use of the critical thermal maxima (CTmax) in ecological contexts to examine the role of thermal tolerance in modulating individual and species-level responses to thermal stress. In Chapter 2, I introduce the concepts and methods associated with CTmax and discuss how this index of upper thermal tolerance has historically been used in fish ecology. The long history of the use of CTmax in addition to the growing state of knowledge in the field of thermal biology has led to the evolution of a series of CTmax protocols. In turn, the variety of applications and protocols introduced a number of concerns regarding the relevance of this metric. After highlighting how recent findings can be integrated in ecological contexts, I 
provide key directions for future research. In Chapter 3, I provide an example of how to further evaluate the ecological relevance of CTmax by adopting a field-based study that compares CTmax of individuals within a population of juvenile semi-anadromous brown trout (Salmo trutta). I further demonstrate how CTmax relates to the decision to migrate, the timing of migration, growth, and predation vulnerability. Investigating how intraspecific variation in upper thermal tolerance leads to differences in fitness by mediating life-history strategies provides a foundation to speculate about the potential for rapid adaptation in an era where temperatures are both fluctuating and rising at unprecedented rates. By shaping CTmax into a tool that can be applied to describe upper thermal tolerance in ecological contexts, we will be able to gain further insight on how organisms and species might respond to climate change. Outlining ecological applications of CTmax and directions for future research provides a useful guide to catalyze research towards a progressive direction in a rapidly warming world. 


\title{
Chapter 2: On the ecological relevance of critical thermal maxima measurements for fishes
}

\begin{abstract}
The critical thermal maximum (CTmax) has been used to infer upper thermal tolerance in fishes for over seven decades, yet its ecological relevance remains debated to this day. Although its relative simplicity and common endpoint has made this method a popular choice to explore fish acute upper thermal limits, some methodological considerations are often overlooked. Here, we identify a series of concerns that have limited the interpretation of CTmax in ecological and evolutionary studies. We synthesize evidence to identify limitations and opportunities associated with this technique, focusing on rates of thermal ramping, acclimation, thermal safety margins, performance traits such as swimming ability, and repeatability. Ultimately, CTmax measurements have been particularly useful to identify temperatures at which organisms fail to perform basic behaviours, such as maintaining an upright position. When accounting for the parameters that can influence these thermal limits (such as acclimation and rate of thermal ramping), CTmax measurements can be used to predict responses to thermal stress. These measurements can then be applied for predictive purposes, for example, mitigating the effects of climate change or infrastructure planning on wild fishes. We conclude by using this insight to describe key directions for future research that will further promote the application of CTmax in ecological contexts.
\end{abstract}

\section{Introduction}

In light of changing global climates, there has been a growing demand for research that focuses on expanding our current knowledge regarding the implications of an animal's upper thermal tolerance, with particular attention to ectotherms. Specifically, many recent studies have 
aimed to predict how changing thermal regimes might influence population distribution, abundance, and ultimately, try to anticipate the 'winners or losers of climate change' (Somero 2010; Sunday et al. 2012; Pinsky et al. 2019; Sunday et al. 2019). Abiotic factors play a considerable role in shaping ecosystems, as they characterize critical habitat while concurrently regulating behavioral and physiological functions in animals. When they become unstable, for example with the predicted increase in frequency and severity of heatwaves (Perkins et al. 2012), ectotherms (including fish) can respond by either changing their behavior, for instance, seeking cooler refuges (Jonsson and Jonsson 2009), or acclimate by modulating physiological responses such as switching to anaerobic metabolism (Pörtner 2002), increasing the production of heat shock proteins or modifying transcriptional regulators (e.g. Clark et al. 2008, Jeffries et al. 2014, Jeffries et al. 2016).

The multitude of levels of responses to thermal stress (i.e. behavioural to molecular) has led to an assortment of methods used to measure thermal tolerance. For instance, some studies measure aerobic scope and maximum metabolic rate across a range of temperatures to determine the temperature at which oxygen supply to tissues becomes insufficient to sustain basic functions (Pörtner 2010). Others measure the expression of heat shock proteins (e.g., Jeffries et al. 2014), or performance traits such as swimming speed and growth at various temperatures (Schulte et al. 2011). However, one of the most prevalent responses to thermal stress across taxa is loss of equilibrium (LOE, inability to maintain dorsal-ventral orientation, Lutterschmidt and Hutchison 1997). As such, the use of critical thermal maximum (CTmax) as a proxy for upper thermal tolerance has become dominant in fish ecology over recent years, as researchers acknowledge the urgency to bridge the gap between thermal tolerance and responses to global climate change (Comte and Olden 2017; Deutsch et al. 2008; Sandblom et al. 2016). Historically, CTmax was 
defined as "the thermal point at which locomotory activity becomes disorganized and the animal loses its ability to escape from conditions that will promptly lead to its death" (Cowles and Bogert 1944). The simplicity of measuring CTmax, along with consistent behavioural responses at upper thermal limits across diverse taxa, have made CTmax a popular choice in fisheries science since its inception (reviewed in Lutterschmidt and Hutchison 1997). In fact, CTmax assisted in the establishment of regulatory guidelines to manage thermal pollution from anthropogenic sources (e.g. Holland et al. 1974). Since then, the broad definition of CTmax was refined to address ambiguities, specifically by incorporating an endpoint, which is typically loss of equilibrium (LOE) or the onset of muscle spasms, as well as the requirement for acute and constant (i.e. $0.3^{\circ} \mathrm{C} \mathrm{min}^{-1}$ ) thermal ramping (Cox 1974; Becker and Genoway 1979; Hutchison 1961; Hutchison 1976; Lutterschmidt and Hutchison 1997; Morgan et al. 2018).

Given the evolution over time of how CTmax has been defined, the literature includes an array of different methods used to derive empirical estimates of CTmax. Inconsistencies across studies have led to much debate regarding the ecological relevance of CTmax, notably concerning the rate of thermal ramping which often does not reflect natural conditions (see Lutterschmidt and Hutchison 1997; Bates and Morley 2020). Several studies claim that sublethal effects arising from prolonged exposure to thermal stress can constrain geographic distribution limits, as it can reduce growth, fecundity, and other fitness related proxies (Pörtner et al. 2017; Williams et al. 2016). While this may be a valid hypothesis for many ectotherms, upper thermal tolerance estimates in the form of CTmax are derived from acute thermal challenges. As such, CTmax only aims at predicting tolerance to acute warming events. However, understanding how CTmax estimates relate to, or are influenced by, sub-lethal effects (reduced growth, fecundity, reflexes) induced by prolonged exposure to warming could provide insight 
regarding the applicability of CTmax as a measure to predict the geographic distribution of species based on environmental temperature.

Much has been written about CTmax, but here, we focus specifically on assessing the ecological relevance of CTmax for fishes. We first review past and current applications of CTmax in fish ecology, a field which includes a rich literature base and more standardized protocols in comparison to other taxa (e.g., sedentary invertebrates). We also review important considerations for measuring and using CTmax in ecologically-relevant ways, and address how issues that arise during experimental assays of CTmax can be avoided. We then highlight how CTmax research can be integrated as a tool to describe individual, population, community and ecosystem-level responses to a progressively warming and more variable environment, and conclude by identifying key directions for future research.

\section{The history of CTmax: Past applications and limitations}

The study of thermal tolerance in fishes has been a topic of interest since the nineteenth century, as researchers notice and attempt to describe the effects of temperature in relation to fish (e.g., Davy 1863, Carter 1887). However, it was not until 1944 that Cowles and Bogert first outlined the use of CTmax protocols to describe thermal limits in ectotherms. Since then, the use of CTmax in ecology increased rapidly - from attempting to understand what temperatures might impair species performance, to forecasting global species redistribution in response to climate change. As novel applications emerged, Cowles and Bogert's original definition of CTmax (1944) evolved to include a stricter set of criteria. In 1974, Cox investigated the effects of three heating rates on the CTmax of bluegill (Lepomis macrochirus) and proposed a more widely accepted definition that defines CTmax as the "arithmetic mean of the collective thermal points at which locomotory activity becomes disorganized, and the animal loses its ability to escape 
from conditions that will promptly lead to its death when heated from a previous acclimation temperature at a constant rate just fast enough to allow deep body temperatures to follow environmental temperature without a significant time lag” (Cox, 1974). This definition emphasized the importance of heating rates, the cumulative effect of thermal ramping on individuals, and the significance of acclimation periods.

Validating thermal ramping rates, specifying size ranges of fish, and including a defined endpoint, presented a set of unambiguous instructions that catalyzed the popularity of CTmax as a method to evaluate and infer upper thermal tolerance in a variety of fishes. In 1979, Becker and Genoway adapted this definition by providing specific guidelines to standardize rates of thermal ramping and to use loss of equilibrium as an endpoint. Becker and Genoway (1979) also acknowledged that excessively slow heating rates might allow experimental animals to acclimate during experimental trials, while rapid heating rates might result in a time lag between deep body and water temperatures. They stated that internal temperatures of small ectotherms would respond to changes in environmental temperature more rapidly than larger conspecifics. They suggested using a rapid heating rate of $0.3^{\circ} \mathrm{C} \mathrm{min}^{-1}\left(18^{\circ} \mathrm{C} \mathrm{h}^{-1}\right)$, so that internal temperatures of both large and small organisms would parallel those of the external environment, a crucial component to estimating CTmax. They further suggested that small or juvenile fish of uniform size should be used in CTmax trials to optimize practicality. Because CTmax estimates can be obtained from a variety of ectotherms, endpoints that mark CTmax differ across taxa. For fish, Becker and Genoway (1979) suggested using loss of equilibrium (LOE) as a prescribed endpoint for CTmax trials, LOE being when the organism loses the ability to right itself and maintain its normal position. 
However, in 1997, Lutterschmidt and Hutchison reviewed 388 publications that used CTmax to infer upper thermal tolerance in a variety of taxa. They found that investigators used different CTmax protocols despite previous efforts to standardize methods. For example, some investigators used heating rates between $1.0{ }^{\circ} \mathrm{C}$ day ${ }^{-1}$ to $5.0^{\circ} \mathrm{C} \mathrm{h}^{-1}$ that could have allowed acclimation to occur during CTmax trials, as these ramping rates may provide sufficient time for organisms to modulate underlying physiological responses related to thermal stress, and thus altering CTmax estimates (Lutterschmidt and Hutchison 1997). They also observed high heating rates of $3.8^{\circ} \mathrm{C} \mathrm{min}-1$ that are expected to cause significant time lags between the internal body temperature of the study organism and the water. Lutterschmidt and Hutchison even found that some researchers failed to disclose the heating rate used altogether. These findings suggest that the importance of heating rate and potential for acclimation have often been ignored (at least up to 1997), which may in turn limit the interpretability of the results.

While there may be benefits to using a prescribed ramping rate, recent studies have instead advocated for the disclosure and validation of a selected ramping rate when conducting CTmax assays on particular species (Jutfelt et al. 2019). Several papers measured significant temperature lags between water and deep tissue in a variety of species, including zebrafish (Morgan et al. 2018), perch (Sandblom et al. 2016), and cod (Jutfelt et al. 2019) using different ramping rates. If all studies performing CTmax assays used a uniform $0.3^{\circ} \mathrm{C} \mathrm{min}^{-1}$, unrealistically high thermal limits may be estimated for larger fishes due to large thermal inertia in relation to body surface area (Jutfelt et al. 2019). Correcting the rate of thermal ramping to account for fish size or morphological differences could therefore provide better representations of thermal limits in fish. 
To date, and despite the widespread use of CTmax as an indicator of upper thermal tolerance, little is known about the underlying physiological mechanisms (or combination of mechanisms) that give rise to this critical endpoint (e.g., Ern et al. 2016, 2017, Jutfelt et al. 2019, Wang et al. 2014). This knowledge gap, among many others, might partially explain why researchers have opted to use different methodologies. For instance, morphological or physiological differences in study organisms could alter the LOE response and lead to over- or underestimated CTmax values. Fish of different age classes could respond differently to thermal ramping due to past thermal exposure; previous exposure to thermal extremes, or lack of extremes, has been shown to alter CTmax estimates (Morgan et al. 2018). CTmax can differ between sexes as well as across populations, and vary depending on fish size and diet (Kumar et al. 2014; Zhang and Kieffer 2014; Gomez et al. 2019; McKenzie et al. 2020; O’Donnell et al. 2020). Some studies have found that CTmax may also vary with environmental variables such as pH, salinity, or dissolved oxygen concentration (e.g., Ern et al. 2016, Madeira et al. 2016, Potts 2020), while others show negligible effects of some abiotic factors (Clark et al. 2017). Some opt to alter the heating rate to mimic more ecologically realistic challenges (e.g., Mora and Maya 2006, Vinagre et al. 2015), while others attempt to obtain simultaneous measurements such as heart rate, metabolic rates or even take blood samples (e.g., Ekström et al. 2016). In other contexts, adopting a specific heating rate presents logistical constraints where researchers may not be able to heat the water at the typical $0.3{ }^{\circ} \mathrm{C} \min ^{-1}$. Although CTmax is often perceived as a simple method to infer thermal tolerance, complex interactions exist when the experimental design deviates from these fundamental concepts. In addition, thermal limits are inherently linked to the environment, morphology, genetics, and physiology, presenting confounding effects that have yet to be fully elucidated. In the following section, we present a series of questions 
regarding CTmax and its ecological relevance, and further provide considerations that should be incorporated into future studies in an effort to increase the applicability of CTmax in fish ecology.

\section{Questioning the ecological relevance of CTmax as a measure of upper thermal tolerance}

\section{Why is the rate of thermal ramping so important when designing CTmax experiments?}

When considering whether CTmax is an ecologically-relevant measure of upper thermal tolerance in fish, one of the most common criticisms is directed at the use of rapid rates (degrees per minutes or hours) of thermal ramping that are rarely observed in the wild (e.g., Terblanche et al. 2007, Chown et al. 2009). The diversity of protocols used to estimate CTmax highlights the importance of time in mediating responses to thermal stress, yet this aspect of thermal tolerance is often ignored in the explanation of hypotheses developed to explain physiological and biological limits (see Bates and Morley 2020; Lefevre et al. 2021). During very fast rates of warming (seconds or minutes) organisms respond to thermal stress by modulating resistance pathways that include increasing the production of heat shock proteins (Clark et al. 2008) or switching to anaerobic metabolism (Pörtner et al. 2002). Alternatively, fish can respond by changing their behaviour, such as seeking cool refuges, or ceasing to feed and digest (Bates and Morley 2020). More specifically, CTmax may reflect the tolerance of immediately critical organs, such as the brain and heart (Ekström et al. 2018; Jutfelt et al. 2019). When thermal ramping occurs more slowly (hours to months), organisms can undergo acclimation, which refers to changes in biochemical pathways and molecules that allow for a new stable physiological state (Bates and Morley 2020). Thus, CTmax is a better estimate for how well organisms can alter resistance rather than acclimatory or adaptive pathways when faced with acute thermal shock, 
and therefore requires thermal ramping rates that alter temperatures by degrees per minute or hour (Bates and Morley 2020).

Although the physiological mechanisms underlying LOE in fishes are not well understood (e.g., Ern et al. 2016, 2017, Jutfelt et al. 2019, Wang et al. 2014), it has been hypothesized that different biological pathways are involved in coping with acute versus chronic thermal stress (Peck 2011; Bates and Morley 2020, Lefevre et al. 2021). For instance, chronic thermal stress (days, weeks, months) can be described by responses such as cessation of feeding, decreased growth rates, or increased predation vulnerability. On the other end of the spectrum, acute thermal stress might refer to short term (minutes to hours) tolerance of immediately critical organs involved in maintaining basic bodily functions. Despite this possibility, some researchers argue that CTmax should be estimated using more realistic heating rates that have greater ecological relevance (e.g., Mora and Maya 2006, Vinagre et al. 2015).

Following a recommended acute rate of thermal ramping to evaluate CTmax is critical for two main reasons: 1) it controls for discrepancies in temperatures between the water and the internal body of the fish, and 2) it prevents the modulation of physiological or biochemical pathways involved in inducing acclimation responses (Becker and Genoway 1979; Lutterschmidt and Hutchison 1997; Beitinger et al. 2000; Mora and Maya 2006). Physiological responses to slower or chronic rates of thermal ramping have been found to vary, with some studies claiming that acclimation occurring during trials leads to overestimation of CTmax (Elliott and Elliott 1995; Beitinger et al. 2000). Others suggest that prolonged exposure to higher temperatures leads to cumulative thermal stress and lower thermal tolerance (Terblanche et al. 2007; Rezende et al. 2014). Given these contrasting findings, it is difficult to conclude whether slower rates describe the same processes involved in thermal tolerance as acute rates in wild fish. 
Indeed acclimation time varies among species (Lutterschmidt and Hutchison 1997; Chung 2001; Vinagre et al. 2015), while lag time (i.e., time for the body temperature to reflect the water temperature) depends on the surface area-to-volume ratio, which is also species-specific and varies with ontogeny (Stevens and Fry 1974). For these reasons, the effects of thermal ramping rates on CTmax and consequently, the ecological relevance of these rates, could vary among species. However, slower (degrees per day) or chronic heating rates ultimately measure a different aspect of thermal tolerance, because CTmax specifically refers to a response derived from an acute thermal stress challenge (Lutterschmidt and Hutchison 1997). Using the term CTmax to describe thermal tolerance derived using slow/chronic rates of thermal ramping adds variation to CTmax values reported in the literature, and hinders the detection of patterns and efforts to use CTmax within an ecological context.

Although it is important to acknowledge that such rapid rates of thermal ramping may rarely occur in the wild, some studies propose that survival during short-duration heat waves (from hours to days) is most important in determining thermal limits (see Åsheim et al. 2020). Fish typically seek their preferred temperature (Larsson 2005; Nivelle et al. 2019), but this may depend on habitat characteristics and species biogeography (Alfonso et al. 2020). Although many fish inhabiting thermally stable habitats do not typically encounter fast heating rates, fish can experience rates similar to those used in CTmax protocols under certain conditions, such as in the intertidal zone, near hydrothermal vents, or if they move through a thermocline (Bates and Morley 2020). Even though fast rates of heating often overestimate functional thermal tolerance (Becker and Genoway 1979), several studies have found evidence that CTmax estimates are highly relevant. For example, global distributions of species have been correlated to CTmax estimates (Payne et al. 2021; Sunday et al. 2012), and mass mortality events of ectotherms have 
been caused by thermal shock (e.g., Wegner et al. 2008; Vertessy et al. 2019; Genin et al. 2020, Finnegan et al. 2012, Penn et al. 2018). The Climate Extremes Hypothesis (Janzen 1967; Gaston et al. 2009; Sunday et al. 2019) states that the evolution of broader thermal tolerance would occur more frequently in species that inhabit environments with more variable temperatures. In the wild, temperature fluctuations tend to follow seasonal or diurnal patterns, where latitudinal gradients often dictate the magnitude of variation observed (Wang and Dillon 2014). The range of temperatures a species or population experiences largely depends on geographic location; in temperate environments, the temperatures experienced can fluctuate from cold (below zero) to hot $\left(30^{\circ} \mathrm{C}+\right)$ depending on seasons, whereas fish in tropical environments will generally experience less variation. The Climate Extremes Hypothesis further predicts that extreme thermal events, even if they do not occur frequently, are key in selecting for thermal tolerance (Pither 2003; Sunday 2019). According to this hypothesis, there should be a positive correlation between heat tolerance indicated by CTmax and the frequency of heat waves. The increased prevalence of heat waves predicted to occur in the near future (IPCC 2014, 2021) could therefore act as a force for directional selection to occur, providing a mechanism for evolutionary rescue from changing thermal conditions. Some studies have demonstrated that populations can function and perform well near their lethal temperature limits (Morgan et al. 2019; Sandblom et al. 2016). Moreover, rapid increases in temperatures can be more influential than endpoints when predicting survival in fish, because the stress response induced during acute thermal ramping increases pathogenrelated mortality (Alfonso et al. 2020; Genin et al. 2020).

Slower rates of thermal ramping (hours to days) may be more reflective of natural thermal challenges, and thus more likely to shape responses of fishes to warming climates (Vinagre et al. 2015, Bates and Morley 2020). However, a recent study by Åsheim et al. (2020) demonstrated a 
positive correlation between rapid warming tolerance and slow warming tolerance in zebrafish. Whether upper thermal tolerance is better characterized by acute or chronic warming summarizes two contrasting views on how organisms respond to warming climates, both of which consider time to be an important dimension of temperature (see Waldock et al. 2018). Upper thermal tolerance derived from chronic warming focuses on how organisms respond to thermal stress over longer time scales, where sub-lethal effects on fitness traits such as growth, swim capacity, immune function, and fecundity, characterize the way organisms respond to warming. In contrast, upper thermal tolerance derived from acute warming focuses on short-term responses of immediately critical organs. The correlation between responses involved in survival during both acute and chronic warming, supports the ecological relevance of CTmax and associated protocols for rapid thermal ramping (Åsheim et al. 2020). Given this finding, both opposing views on how organisms respond to warming could be linked through similar physiological mechanisms, and both hypotheses could provide valuable insight for how selection might occur in response to climate change ( $\AA$ sheim et al. 2020).

In summary, rapid rates of thermal ramping may not always represent conditions in the wild, but upper thermal limits obtained from this approach are still useful because they depict how fish will respond to extreme weather events. Inconsistent rates of ramping in the literature will lead to either the overestimation or underestimation of critical thermal limits, which is why CTmax estimates should be interpreted in the context of the animal's thermal history, as well as the experimental design and protocol that generated the estimate. Given that CTmax is characterized by acute responses to thermal stress (primarily through resistance pathways), researchers should proceed with caution when describing estimates obtained using thermal ramping rates that occurred over longer time scales. These estimates may be underpinned by fundamentally 
different mechanisms that limit thermal tolerance (such as protein denaturation versus oxygen or energy limitations). Thus, despite the apparently higher ecological relevance of chronic warming rates, the term CTmax should only be used when referring to upper thermal limits derived under acute rates of thermal ramping.

\section{How does acclimation influence CTmax?}

Time is important in mediating acclimatory processes, i.e. the changes in biochemical pathways and molecules that allow for a new stable state in response to environmental or treatment conditions (Bates and Morley 2020). A fish is 'acclimated' to its environmental temperature when, for example, the energy allocated towards reproduction is maximized at a particular temperature (Bates and Morley 2020). In CTmax experiments, the term 'acclimation' is often misused to refer to the adjustment period prior to conducting experimental trials (Bates and Morley 2020). In fact, very few CTmax studies actually measure indices of acclimation yet most still claim that organisms were acclimated. Recording measurable changes in energy expenditure from one stable state to the next (i.e., from one temperature to another) might require experimental trials to last several months, rather than a few weeks, depending on the magnitude and rate of environmental change.

Because acclimation is energetically costly (Angilletta 2009), acclimation may only be beneficial when environmental changes are predictable and long-lasting, such as with seasonal cycles (Bates and Morley 2020). However, the increased frequency of heat waves and drastic temperature fluctuations associated with climate change predictions (IPCC 2014) might be too short in duration to allow for full acclimation to occur. Fish that historically have been exposed to variation in thermal conditions could show better acclimation potential, while those that evolved in homogeneous environments may not, given that acclimation is energetically costly 
(Krebs and Loeschcke 1994, Morgan et al. 2019). If energy is allocated towards acclimation, then resources must be diverted away from other functions, including growth, immune function, and reproduction (Krebs and Loeschcke 1994).

Although it is widely accepted that acclimation influences upper thermal tolerance in fish and other ectotherms (Beitinger and Bennett 2000; Schulte et al. 2011; Huey et al. 2012), the underlying physiological mechanisms leading to variability in these estimates remain poorly understood (McKenzie et al. 2020, Lefevre et al. 2021). Studies attempting to determine the temperatures at which fish no longer cope with warming often encounter difficulty in making predictions owing to the effects of acclimation. In some studies, a so-called acclimation period is mentioned, but not confirmed with physiological measurements establishing a new steady state; in others, acclimation is not mentioned at all. Discrepancies in acclimation (i.e., presence, absence, or lack of reporting) have important ramifications on the measured CTmax values, making it difficult to compare results across studies or perform meta-analyses or data syntheses with existing literature (Lutterschmidt and Hutchison 1997).

Generally, acclimation effects in fish can be observed across a large range of temperatures. Acclimation to higher temperatures typically yields higher CTmax values, with values converging towards an asymptote as acclimation temperature increases (Chen et al. 2015; Morgan et al. 2019; McKenzie et al. 2020). Thermal safety margins - the difference between acclimation temperature and CTmax - narrow as fish acclimate to higher temperatures across their thermal range (McKenzie et al. 2020). This pattern indicates that although CTmax is a plastic trait, it follows the principles of 'concrete ceilings' (Sandblom et al. 2016; Morgan et al. 2020). Fish already living close to their upper lethal temperature limits thus have narrow thermal 
safety margins, as they are already living at temperatures near the higher end of their thermal range (see Morgan et al. 2019).

Some studies have already identified populations living close to their upper thermal limits. For example, Morgan et al. (2019) found that wild zebrafish live in environments where temperatures can reach lethal extremes, and that individuals in warmer habitats had higher CTmax, perhaps as individuals have acclimated to different thermal environments. Moreover, Morgan et al. (2019) investigated whether domesticated and wild zebrafish from the same population differed in thermal tolerance, because domesticated fish are reared in environments with constant temperatures and do not experience environmental fluctuations. Since acclimation is energetically demanding, the authors hypothesized that thermally stable environments would lead to lower acclimation capacity in domestic fish, owing to selection against the traits involved in thermal tolerance. However, they found that thermal tolerance was maintained during domestication, suggesting that traits involved in thermal tolerance are highly conserved despite being costly to maintain (Morgan et al. 2019). Moreover, the study detected no difference in CTmax between the domesticated populations, regardless of the duration of domestication. So, despite differences between domesticated and wild populations, laboratory-reared model species can be useful to address certain questions pertaining to thermal tolerance.

The rate at which fish can acclimate to changing conditions will in part determine which species will survive under future climate scenarios (although mobile species may be able to relocate to suitable habitat conditions elsewhere). Fish with a capacity for rapid acclimation, provided energetic reserves are not depleted, may become the 'winners' of climate change (Somero et al. 2009). In fact, adaptation can be accelerated by plasticity (West-Eberhard 2003; Lande 209; Chevin and Lande 2010), which indicates there is some positive genetic correlation 
between acclimation phenotypic plasticity and CTmax. Morgan et al. (2020) quantified the contribution of acclimation to upper thermal tolerance over 6 generations of artificial selection to higher thermal tolerance in zebrafish, and found that the acclimation capacity declined when the populations evolved higher thermal tolerance. These findings show that there is low potential for evolutionary rescue in tropical populations of fish that already live close to their thermal extremes. The effects of acclimation may be more beneficial in populations living in temperate environments where seasonal fluctuations in temperature are more predictable (Wang and Dillon 2014; Morley et al. 2019), although previous studies failed to find a link between plasticity and latitude or seasonality (Gunderson and Stillman 2015).

Future studies attempting to infer responses to climate change should focus on determining acclimation potential in wild populations. Fish are often acclimated to temperatures that reflect optimal temperatures in their natural environment. Pushing acclimation towards higher temperatures when performing CTmax assays will reduce the variability in estimated thermal limits (especially in temperate species) and provide a more accurate prediction of temperature extremes at which fish can survive. Determining rates of acclimation over a range of temperatures in populations of different species will facilitate comparisons of populations living at warm and cool range edges, and also between temperate and tropical species (e.g., Morley et al. 2018). So, to increase the ecological relevance of CTmax estimates for predicting species distribution, fish should be fully acclimated prior to conducting CTmax trials, and this acclimation should be confirmed using reliable measurable indices (e.g., metabolic rate). Conducting CTmax assays on fish that are fully acclimated to various temperatures will provide a range of temperatures over which they can still sustain basic physiological demands while identifying the degree of phenotypic plasticity of acute upper thermal tolerance. Finally, the rate 
of acclimation itself should be reported, as it conveys important information required to understand how fish will survive periods of exposure to supra-optimal temperatures.

\section{How does CTmax compare to other estimates of thermal tolerance to determine thermal performance?}

By definition, CTmax is a critical thermal limit that represents extreme temperatures at which fitness or performance reaches zero (MacMillan 2019); at their CTmax, fishes experience LOE and are unable to escape from conditions that will promptly lead to their death (Cowles and Bogert 1944). However, whether CTmax relates to or predicts various performance traits in fish, such as growth, reproductive success, or swimming ability, is poorly understood. This is partly owing to lack of knowledge about the underlying physiological processes that lead to LOE with acute heat exposure. Understanding the extent to which CTmax relates to performance by exploring questions such as whether fish with higher CTmax swim better in supra-optimal conditions or whether fish with lower CTmax are less likely to forage in warmer waters, will provide further insight into the ecological relevance of CTmax as a proxy for upper thermal tolerance. For example, if fish with higher CTmax consistently perform better than conspecifics in warmer temperatures and fish with lower CTmax perform better at lower temperatures, CTmax could be used as a proxy for thermal performance under, for instance, heat waves or in areas with warm-water discharge.

Several studies have used thermal performance curves (TPCs, Figure 1) as a tool to determine how different species respond to climate change (Dillon et al. 2010; Deutsch et al. 2008; Huey et al. 2012; Sinclair et al. 2016). Thermal performance curves describe the relationship between body temperature and performance in ectotherms (with critical minima at extreme cold and heat, and a peak in performance predicted at intermediate temperatures where 
optimal temperatures are reached). These curves are fundamentally characterized by low performance at critical thermal limits (critical thermal minimum for the lower limit of thermal tolerance, and CTmax), maximal performance at optimal temperature, and a temperature range at which performance remains above 50\% of its maximum (Rezende and Bozinovic 2019). Performance indices include behaviour, life-history traits, and physiological variables in ectothermic organisms (Rezende and Bozinovic 2019). At the whole-organism level, performance traits of interest often include fecundity, growth, metabolic rate, and swimming speed (Schulte et al. 2011). On smaller scales, performance traits may include heart rate, nerve conduction velocity, and enzyme activity. Metrics are typically biological rate processes such as offspring per lifetime, amount of oxygen consumed per unit time, distance traveled per unit time, and enzyme reaction rates (Schulte et al. 2011). The increase in performance as temperatures reach optimal levels are thought to reflect fundamental effects of thermal dynamics on molecular movements, whereas the decrease at supra-optimal temperatures is linked to reversible or irreversible protein denaturation and other temperature-dependent destabilizing effects (Schulte et al. 2011). The shape and breadth of TPCs can vary across levels of biological organization, according to seasonal patterns such as reproduction or migration, with phenotypic plasticity, with geographic location, and across acute or chronic time scales (Schulte et al. 2011; Rezende and Bozinovic 2019). Our understanding of the mechanisms underlying the responses of organisms to thermal stress and how TCPs translate to the success of fish in nature is incomplete, hindering our interpretation of differences in the shape of TPCs (Schulte et al. 2011; Rezende and Bozinovic 2019).

Because CTmax is measured using acute thermal ramping, TPCs generated under equivalently rapid rates of heating provide insight into how CTmax relates to the trait being 
measured (e.g., Rezende et al. 2014, Dowd et al. 2015, Kingsolver and Woods 2016). For example, relating CTmax to short-term performance traits could be done by conducting an experiment during which swimming speed is measured with increasing temperatures until a fish experiences LOE. Such an experiment would help researchers directly relate CTmax to swimming speeds and provide clear insight on how acute thermal stress impacts performance.

Previous studies have attempted to measure swimming speed in relation to temperature increases, though they have ultimately measured different endpoints. Steinhausen et al. (2008) measured $\mathrm{T}_{\text {crit, }}$ the point at which aerobic metabolism transitions to anaerobic metabolism during swim trials where temperature was increased every 30 minutes (Steinhausen et al. 2008). In this case, relating these $\mathrm{T}_{\text {crit }}$ measurements to CTmax measurements on the same individuals using an acute rate of ramping would provide insight on how swimming speed relates to CTmax. More recently, Blasco et al. (2021) investigated whether $\mathrm{CT}_{\text {swim }}$ (the point at which fish are unable to swim) could be used as an alternative to LOE in CTmax experiments. While they attempted to relate $\mathrm{CT}_{\text {swim }}$ to a form of $\mathrm{CTmax}$, they used a slow rate of ramping $\left(1^{\circ} \mathrm{C}\right.$ per 30 minutes $)$ which deviates from the standardized procedure. Similarly to Steinhausen et al. (2008), correlating $\mathrm{CT}_{\text {swim }}$ estimates to $\mathrm{CTmax}$ estimates derived under acute rates of ramping would reveal correlations between swimming performance and CTmax.

Studies have opted to conduct thermal performance experiments over longer time scales to mimic how the degree of thermal stress experienced under prolonged exposure regimes influences key animal response (e.g., reproduction: Deutsche et al. 2008). Indeed, cumulative effects of sub-lethal and long-term temperatures experienced may influence energy balance (Dillon et al. 2010), fecundity and developmental rates (Huey and Berrigan 2001), and ultimately fitness (Rezende and Bozinovic 2019). Upper thermal limits for functional traits are different 
than CTmax. However, CTmax remains useful as an index to compare against these functional traits.

There is also the possibility that CTmax relates to functional thermal performance traits derived under slower rates that are more commonly observed in the wild (degrees per day), since they may share similar underlying mechanisms (Åsheim et al. 2020). Some ectotherms display 'thermal types', where some individuals are consistently cold-tolerant and some are warmtolerant (Goulet et al. 2017a). The notion of 'types' is based on a theoretical framework for studying systems of correlated traits, and considering the links among temperature, metabolism and behaviour. Goulet et al. (2017a) suggested that an individual's thermal type would align with behavioural and life-history types. Cold-type individuals would have a left-shifted TPC, while warm-type individuals would have a right-shifted TPC. Åsheim et al. (2020) tested whether upper thermal tolerance to rapid heating rates correlated to that derived under slow heating rates and growth in zebrafish. Although thermal tolerance to rapid heating did not correlate to growth performance, the findings indicated a correlation between thermal tolerance obtained from rapid and slow rates of warming. Thus, there were individuals with consistently (relatively) higher thermal tolerance, acting as a 'warm-type', and others with consistently (relatively) lower thermal tolerance (Åsheim et al. 2020). The lack of correlation between thermal tolerance derived under rapid heating and growth suggests that acute thermal tolerance has little mechanistic association with growth performance under supra-optimal temperatures. Moreover, this may reflect a very limited scope for a thermal syndrome, though alternative mechanisms of thermal tolerance could be organized in some form of thermal syndrome, as found in reptiles (Goulet et al. 2017a, b; Michelangeli et al. 2018; Åsheim et al. 2020). 
By integrating a combination of conceptual frameworks that attempt to explain the underlying mechanisms of upper thermal limits in wild fish, we will be able to gain a more holistic understanding of individual, population, and species responses to warming. Desforges et al. (2021) attempted to link CTmax to performance indices, such as growth, migration strategy and predation vulnerability (Desforges et al. 2021), but found no evidence that differences in CTmax were associated with variation in performance traits. However, studies that used alternative indices of upper thermal tolerance, such as cardiorespiratory performance traits in different sockeye salmon populations, have identified links with performance traits related to migration difficulty and temperatures experienced in the past (Eliason et al. 2011). Additionally, Chen et al. (2013) measured CTmax in juvenile sockeye salmon from the populations outlined in Eliason et al. (2011), and found CTmax to be higher in populations with greater migration difficulty. This suggests that mechanisms underlying CTmax have ecologically relevant applications, as they are linked to endurance and ability to cope with strenuous challenges. However, these laboratory-reared fish may not accurately represent trends occurring in the wild. Using CTmax to study populations in the field, as described in Desforges et al. (2021) to describe differences in performance across individuals, will further highlight the ecological relevance of acute upper thermal tolerance.

Regardless of the index used to estimate upper thermal tolerance, each method comes with limitations; the physiological mechanisms underlying these responses are complex and cannot be fully described by a single measure (Rezende et al. 2014; Rezende and Bozinovic 2019; Lefevre et al. 2021). Although CTmax is a useful tool to describe tolerance to extreme weather events or explain certain geographic or behavioural patterns, methodological constraints and the degree of uncertainty associated with the physiological mechanisms involved in LOE are major limitations 
to the applicability of CTmax. There is some speculation that immediately critical organs, such as the brain or heart, are responsible for performance decline during acute thermal stress. However, the mechanism by which CTmax relates to the decline of other important organs during longer exposures to sub-lethal temperatures is not likely to be elucidated given current protocols (Lefevre et al. 2021). Organs could fail at similar temperatures, but across different lengths of exposure (Lefevre et al. 2021). Some studies advocate for the use of an ecologicallyrelevant sub-lethal threshold based on fatigue from exercise performance as an endpoint rather than LOE. Recently, Blasco et al. (2020) argued that CTswim, an endpoint that involves fatigue derived from sustained aerobic swimming, provides a more ecologically-relevant sub-lethal threshold for tolerance of acute warming than LOE in fishes (Blasco et al. 2020). The development and testing of such conceptual frameworks that attempt to link CTmax to performance traits will help to predict responses to climate change, as well as exploring the physiological responses of organs involved in the response to thermal stress.

\section{What are thermal safety margins and how can CTmax be used to determine them?}

Thermal (heat) safety margins generally refer to an excess of warm thermal tolerance (Sunday et al. 2014), and they have been used to predict the degree of physiological stress caused by warming (Pinsky et al. 2019). Although heat safety margins do not predict how species will respond to warming, these margins allow comparisons of how different species will tolerate increasing temperatures (Pinsky et al. 2019). A variety of different approaches have been developed to quantify thermal safety margins depending on the study objectives. For example, some studies described them as the difference between acute upper thermal tolerance and acclimation temperature (McArley et al. 2017; McKenzie et al. 2020). Huey et al. (2012) made significant contributions to this field and stated that the physiological impact of warming 
depends on the extent of an organism's ability to tolerate high body temperatures above thermal optimum (Huey et al. 2012). Pinsky et al. (2019) measured thermal safety margins as the difference between the acute upper thermal limit of a species and the extreme hot hourly body temperature of the species in the coolest microhabitat that is available. For insects, Deutsche et al. (2008) refer to thermal safety margins as the difference between an organism's thermal optimum for reproduction and its current climate temperature. Vinagre et al. (2019) calculated them as the difference between CTmax and maximum habitat temperature, or, for future safety margins, maximum habitat temperature $+3^{\circ} \mathrm{C}$. Comte and Olden (2017) used the difference between CTmax and the mean temperature of the warmest month experienced by various marine fishes across their range. There appear to be many different ways of inferring sensitivity to warming, but most studies have used CTmax as an index for upper thermal tolerance. However, given that CTmax is influenced by acclimation temperatures, it is important to note that these upper boundaries can be somewhat flexible - especially in temperate species that are, in most cases, not living near their thermal extremes.

There is also the issue that species can occupy broad geographic ranges, where specific populations are acclimated to different temperatures, experience different degrees of thermal fluctuation, and may in turn exhibit different levels of phenotypic plasticity and CTmax owing to population-level adaptation to local environmental conditions (Comte and Olden 2017). Attempts have been made to account for this variability while modelling species distribution and predicting responses to climate change (e.g., Sunday et al. 2014, Comte and Olden 2017, Pinsky et al. 2019). To investigate climate vulnerability in marine and freshwater fishes, Comte and Olden (2017) compiled a database of 485 species with a total of 2,722 thermal challenges (including both static and dynamic) at various acclimation temperatures. Although several 
assumptions had to be made to derive these estimates, the authors found that upper thermal tolerance varied considerably according to geography and taxonomy, with the highest upper thermal limits observed near the equator in both freshwater and marine fish. They fitted a model between upper thermal limits and the temperature of acclimation to account for the fact that thermal limits vary with acclimation history, and that temperatures used in these thermal challenges are not always representative of species' thermal habitat (see Comte and Olden 2017). The model was used to derive $30 \mathrm{CTmax}$ values for each species at the mean summer temperatures experienced across their range while accounting for plasticity in thermal responses. Despite having higher upper thermal tolerances, tropical fishes are the most impacted by warming climates as they already live close to their thermal limits. Under future climatic conditions, fish inhabiting northern high-latitude freshwater basins are projected to display the most sensitivity to warming (Comte and Olden 2017).

Similarly, Pinsky et al. (2019) used CTmax to make inferences about the vulnerability to warming of marine and terrestrial organisms. The authors compiled a dataset on 88 marine and 318 terrestrial species, and corrected upper thermal tolerance estimates by incorporating acclimation temperature in their models. Considering the evidence for greater acclimation capacity in marine than terrestrial organisms, and the idea that acclimation capacity does not vary strongly across latitude (Gunderson and Stillman 2015), the authors opted to use average summer temperatures to adjust upper thermal tolerance estimates. They further compensated for the effects of acclimation by using a mean acclimatization response ratio, which reflects the degree to which thermal limits increase or decrease with changes in the acclimatization temperature (Pinsky et al. 2019). However, one of the limitations of this approach is that acclimation responses are non-linear and acclimation to lower temperatures requires less energy 
expenditure than acclimation to temperatures closer to upper thermal limits (Morgan et al. 2019). Moreover, there are also major species differences, for example, gobies acclimate very poorly (Christensen et al. 2021) while many other species acclimate relatively well (Morgan et al. 2019).

Thermal safety margins can be over-estimated (i.e. too broad) if the experimental data used were compiled with arbitrary acclimation temperatures (i.e. temperatures that are not ecologically-relevant but rather used for logistical purposes), which frequently occurs in CTmax studies (Sunday et al. 2014). Tropical species experience little fluctuation in temperature (Wang and Dillon 2014) and are acclimated to higher temperatures closer to their upper thermal limit, as such, narrow safety margins are often extrapolated. Recently, Payne et al. (2021) found that tropical species actually show broader heating tolerances at a given acclimation temperature compared to temperate species, although they also show smaller heating tolerances at higher temperatures. Although thermal safety margins appear greater in tropical species acclimated to the same optimal temperature as a temperate species, temperate species show greater capacity to cope with increases in temperature than tropical species. However, to make similar comparisons with temperate species, CTmax values would need to be determined using the warmest temperatures these species experience in the wild, across their geographical range. Methodological variation in CTmax protocols can therefore lead authors to make incorrect inferences on thermal safety margins and species responses to warming when CTmax values are not adjusted according to the highest acclimation temperatures experienced in the wild. Many studies that use CTmax attempt to answer specific questions; they may thus tailor methods to investigate particularities of organisms within their study, without standardizing their results for inclusion in meta-analyses or data syntheses. It is essential for future studies that compare 
interspecific upper thermal tolerance in the form of CTmax to acknowledge the importance of and report on methodological differences in their attempts to determine thermal safety margins and model responses to warming.

\section{Is CTmax repeatable, and what does that tell us about heritability and adaptive potential?}

In ecological and evolutionary research, repeatability tends to be positively correlated with heritability (Boake 1989; Dohm 2002; Bell 2009). In fact, Boake (1989) suggests that repeated measures allow researchers to make inferences about rates of evolution, because both the rate of evolution and the magnitude of heritability are constrained by repeatability. Thus, the repeatability of thermal tolerance metrics has been used as an approach to make inferences about the adaptive potential of species in relation to climate change or other environmental changes (Killen et al. 2016; Morgan et al. 2018). To assess the adaptive capacity of a population, there must be phenotypic variation in the trait of interest, the trait must be heritable, and there must be selection for the trait. Here, heritability is defined as the ratio between the amount of additive genetic variance and the amount of phenotypic variance within a population (Falconer and Mackay 1996). A heritability value close to 1 implies that almost all of the variability in a trait comes from genetic differences, with very little contribution from environmental factors. Repeatability, on the other hand, shows the consistency of an individual's performance over longer timescales, by quantifying the proportion of total variation of a trait that is due to differences between individuals (Dohm 2002; Bell 2009). Within-individual repeatability refers to the degree of consistency in reproducing a trait of interest over time in an individual subject (i.e., temporal stability of a trait), whereas between-individual repeatability accounts for the proportion of total variation for a trait within a population (Killen et al. 2016). 
Estimating the repeatability of individual CTmax in a variety of species or taxa, over both short and long timescales, across life stages, under a range of ecologically-relevant environmental conditions, and when exposed to stressors, would provide insight into the potential for evolutionary responses under a warming climate. Repeatability of CTmax would suggest that this trait is, at least partially, the result of genetics, thus providing a mechanism upon which natural selection can occur. For example, fish with consistently low CTmax under a wide range of environmental conditions might be more susceptible to thermal stress than conspecifics with higher CTmax, potentially resulting in lower lifetime fitness or premature death under extremely high temperatures. Despite the use of CTmax since the 1940s, researchers have only recently started to explore how repeatable CTmax estimates are for individual organisms, and under what conditions. Repeatability of CTmax estimates would support the ecological relevance of CTmax.

Morgan et al. (2018) investigated the repeatability of CTmax in zebrafish (Danio rerio) and found the repeatability coefficient to be 0.45 once effects of heat hardening were taken into consideration. Similar to heritability coefficients, repeatability coefficients can range from zero to one, with values closer to one representing greater repeatability. 'Heat hardening' refers to an acclimation effect where the response to warming is altered owing to previous exposure to acute thermal stress, likely through changes in physiological and biochemical pathways that increase resistance to future heat exposure. The findings of Morgan et al. (2018) demonstrated that although CTmax seems to be repeatable, it is unclear how much of the repeatability can be associated with environmental factors and how much can be associated with genetics. The genetic variability underlying thermal tolerance provides a basis for natural selection to occur, allowing populations to evolve or alter their thermal tolerance. This could have important 
benefits for range expansion or species redistribution, and improved coping with global climate change (Morgan et al. 2018). While these findings are relevant for short timescales (days to weeks), others have found evidence of repeatability in fish over longer timescales (repeatability coefficient of 0.48 for months to one year in Salvelinus fontinalis, 0.43 for six weeks in Poecilia reticulata; O'Donnell et al. 2020 and Grinder et al 2020, respectively). All repeatability studies found evidence of heat hardening. These findings provide further support for CTmax being a repeatable trait within individuals of diverse species. Other studies that used alternative methods to measure upper thermal tolerance in fishes also have found evidence of heritability (e.g., Perry et al. 2005, Anttila et al. 2013, Muñoz et al. 2014, Muñoz et al. 2015), suggesting that thermal tolerance is heritable (at least in part), whether it be estimated using CTmax or other methods. Although acute upper thermal tolerance likely has a genetic component, clear evidence of a correlation between repeatability and heritability in CTmax under natural conditions is still lacking. Because heritability is influenced by phenotypic variance, heritability could decrease under natural conditions owing to increased individual phenotypic plasticity (Nussey et al. 2007; Dingemanse et al. 2010; Killen et al. 2016). A meta-analysis by Bell et al. (2009) found that several behavioural traits that were repeatable often differed among age classes, across sexes (also reported in O'Donnell et al. 2020), and between field and laboratory studies. Many factors can elicit plasticity in CTmax estimates, including differences in life stages (e.g., Recsetar et al. 2011, Illing et al. 2020), diet (Isaza et al. 2019), water quality (e.g., Liddy and Wissing 1988, Sardella et al. 2008; Ern et al. 2016; Potts 2020), habitat (Rodgers et al. 2019), reproductive stage, and social status (LeBlanc et al. 2011; Bard 2020). Chronic stress in fish also impaired responses to thermal stress (e.g., LeBlanc et al. 2011; Claireaux et al. 2013). These observations raise the question of whether CTmax is as repeatable, and therefore heritable, in the wild, as it 
appears to be under controlled laboratory conditions, particularly because environmental effects could mask genetic differences (Bell et al. 2009).

Repeatability is therefore context-dependent and under particular conditions, a trait with high repeatability, like thermal tolerance, can effectively impact ecological performance and fitness (Claireaux et al. 2013; McKenzie et al. 2020). To explore these links under more natural yet controlled conditions, Claireaux et al. (2013) exposed European seabass to stressful conditions (oil or chemically-dispersed oil) in mesocosms, and found evidence of repeatability in thermal responses. The authors used a different approach than CTmax, but their study still provides insight into the relationship between repeatability and environmental stress. A control group, where fish were not exposed to oil or chemical dispersant, revealed repeatable measures of time to loss of equilibrium (TLOE), with a large degree of between-individual variation (Claireaux et al. 2013). After a month of exposure to oil or a chemically-dispersed oil, individuals that died earlier were found to have lower thermal tolerance. In this case, tolerance to these thermal challenges predicted survival, a proxy for Darwinian fitness. The between-individual trait variation along with strong selective pressures led to a higher frequency of thermally-tolerant individuals, promoting directional selection.

More research is required to better understand the links between repeatability and heritability, with particular consideration for differences between wild and laboratory conditions (Killen et al. 2016). The potential for environmental factors and anthropogenic stressors to shape CTmax should not be neglected when making inferences on the adaptive potential of populations to changing climate. Though several studies found high repeatability coefficients for CTmax, these results should be interpreted with caution because the degree to which environmental factors impact CTmax remains largely unknown. Repeatability often sets the upper limit to 
heritability (Falconer 1981; Dohm 2002; Dochtermann et al. 2015; Killen et al. 2016), and Morgan et al. (2018) found repeatability estimates to be greater than the heritability estimates from previous studies (e.g., Doyle et al. 2011, Meffe et al. 2011, Baer and Travis 2000). However, when there are significant genotype-environment interactions, repeatability may not always set upper boundaries for heritability (Dohm et al. 2002).

Another interesting question is whether warming rates in natural settings will be sufficient to promote evolution for higher CTmax at a rate that will allow populations to cope with climate change. By artificially selecting for CTmax over six generations of zebrafish under laboratory conditions, Morgan et al. (2020) recently showed that although there was potential for adaptation of upper thermal tolerance, the rates of adaptation were slow. The study found evidence of both up-selection and down-selection evolution of upper thermal tolerance, with up-selection being significantly slower $\left(0.04 \pm 0.008^{\circ} \mathrm{C}\right)$ and reaching an upper limit (Morgan et al. 2020). These findings imply that natural selection will be insufficient to generate rapid change, suggesting low potential for evolutionary rescue. More studies are needed to assess the potential for evolutionary rescue in a range of species if we are to adequately predict whether species will cope with global climate change.

In summary, genetic differences are likely present within populations, but aspects of environmental change could mask the effects of genetic differences, and thus the extent to which these traits can be targeted for selection (Killen et al. 2016). Making inferences on the potential for adaptive evolution in response to climate change based solely on the repeatability of CTmax could yield misleading predictions. When considering correlations among repeatability, heritability, genetics, and adaptive potential, it is critical to consider the influence of external factors on the physiology underlying CTmax. Individual phenotypic plasticity is context- 
dependent and changes over time, hindering repeatability in natural settings (Nussey et al. 2007; Dingemanse et al. 2010; Claireaux et al. 2013; Killen et al. 2016). We suggest that future studies focus on measuring the repeatability of CTmax under a range of environmental conditions.

\section{Conclusion}

Assessing thermal tolerance has been a priority in the field of ecology, in effort to predict whether species will survive global changes in climate. Understanding upper thermal limits is not only relevant to climate change, but also to infrastructure planning, such as electricity generation, industry, and stormwater management (e.g. Turko et al. 2020). This review advances the debate surrounding the ecological relevance of CTmax, and informs those that have recently been introduced to the field of thermal biology of fishes. Although CTmax has been criticized as an overly simplistic way of measuring thermal tolerance, it remains an integrative metric with comparable endpoints across populations and taxa. Moving forward, the use of a standardized protocol will be necessary to homogenize data and further advance the field. In particular, a standardized protocol could be used to detect patterns across and within species, a task currently made difficult by the variability in protocols. When performed adequately with rapid rates of heating and accounting for the potential effects of external factors, CTmax has been shown to be repeatable, comparable to other metrics of thermal limits obtained under slow rates of warming, and ecologically relevant. However, CTmax should not be considered a 'silver bullet'; our understanding of the physiological mechanisms that lead to CTmax, LOE in particular, remains incomplete. Instead, we argue that CTmax is a tool that should be used in combination with other indices of thermal tolerance to produce a more holistic description of thermal tolerance in fish. Field-based studies that integrate multiple approaches to measure thermal tolerance and performance in wild fish will likely yield the greatest insight. Our incomplete understanding of 
the physiological responses that underlie thermal stress has resulted in 'thermal tolerance' being only loosely defined. Temperature varies across time and space and as such, predictions are reliant and complex multidimensional variation. Perhaps unconsciously, researchers have used the broad definition of thermal tolerance with widely different approaches, often not comparable to one another. There are now many perspectives on what might constitute the 'best' index of thermal tolerance. The most relevant approach will likely require careful contextualization to ensure study objectives match the index selected, which will involve synthesizing mechanistic explanations, since thermal stress acts on multiple levels of biological organization and differs across time scales.

\section{Acknowledgements}

Funding for this project was made possible through an NSERC Discovery Grant awarded to S.J.C., K.M.G., and A.E.B. In addition, K.B.G. is supported by the Villum Foundation. 


\section{Tables}

Table 2-1. Summary of considerations for making CTmax more ecologically relevant.

\begin{tabular}{|c|c|c|}
\hline Points of interest & $\begin{array}{l}\text { Issues with the current } \\
\text { standing }\end{array}$ & $\begin{array}{l}\text { Recommendations to make } \\
\text { CTmax more ecologically } \\
\text { relevant }\end{array}$ \\
\hline Thermal ramping & $\begin{array}{l}\text { - Lack of consistent thermal } \\
\text { ramping across studies } \\
\text { - Slow ramping does not } \\
\text { actually measure CTmax } \\
\text { - Thermal ramping rate } \\
\text { sometimes not reported }\end{array}$ & $\begin{array}{l}\text { - Disclosure and validation of } \\
\text { rate of thermal ramping } \\
\text { - Rate of thermal ramping must } \\
\text { be acute and ramped at a } \\
\text { consistent rate until LOE is } \\
\text { observed }\end{array}$ \\
\hline Acclimation & $\begin{array}{l}\text { - Fish are not always acclimated } \\
\text { - Details of acclimation are not } \\
\text { always disclosed } \\
\text { - CTmax is not comparable due } \\
\text { to choice of acclimation } \\
\text { temperature }\end{array}$ & $\begin{array}{l}\text { - If attempting to compare } \\
\text { CTmax endpoints to other } \\
\text { studies for predictive purposes, } \\
\text { ensure fish are fully acclimated } \\
\text { to high temperatures using } \\
\text { measurable indices. } \\
\text { - Acclimate fish to different } \\
\text { temperatures to determine the } \\
\text { degree of phenotypic plasticity } \\
\text { - Report the rate of acclimation }\end{array}$ \\
\hline Thermal safety margins & $\begin{array}{l}\text { - Different ways of measuring } \\
\text { according to research question }\end{array}$ & $\begin{array}{l}\text { - Use a standardized approach } \\
\text { that considers differences in } \\
\text { protocols } \\
\text { - Ensure metrics are comparable }\end{array}$ \\
\hline Measures of repeatability & $\begin{array}{l}\text { - Laboratory derived } \\
\text { repeatability estimates do not } \\
\text { represent those that would be } \\
\text { derived in the wild } \\
\text { - No evidence for evolutionary } \\
\text { rescue }\end{array}$ & $\begin{array}{l}\text { - Perform field CTmax assays } \\
\text { on wild fish to determine } \\
\text { whether CTmax is repeatable } \\
\text { under natural conditions } \\
\text { - Develop a greater } \\
\text { understanding of underlying } \\
\text { mechanisms involved in LOE }\end{array}$ \\
\hline Thermal performance & $\begin{array}{l}\text { - Few links between CTmax and } \\
\text { performance indices } \\
\text { - Some frameworks are more } \\
\text { useful to measure functional } \\
\text { performance than CTmax }\end{array}$ & $\begin{array}{l}\text { - Continue exploring the } \\
\text { possibility of having a 'thermal } \\
\text { type' and how these relate to } \\
\text { performance traits } \\
\text { - Identify correlations between } \\
\text { CTmax and alternative indices } \\
\text { of thermal tolerance }\end{array}$ \\
\hline
\end{tabular}




\section{Figures}

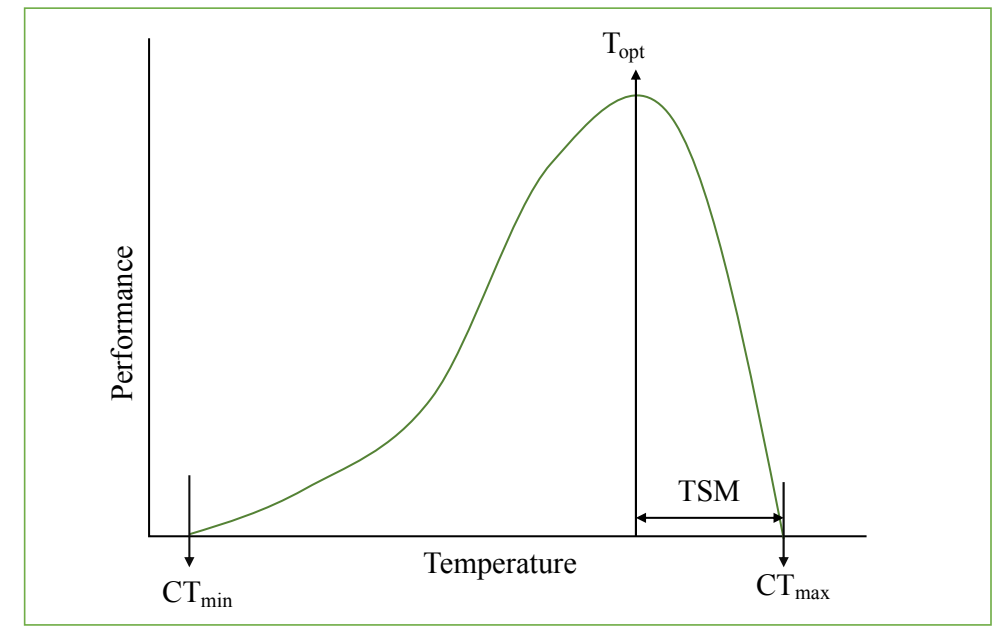

Figure 2-1. Thermal Performance Curve. Conceptual diagram of thermal performance curves (TPC), with critical thermal minima (CT $\min )$ and critical thermal maxima $\left(\mathrm{CT}_{\max }\right)$ represented where performance approaches zero, the thermal optima $\left(\mathrm{T}_{\mathrm{opt}}\right)$ represented at a temperature where the organism exhibits maximal performance, and thermal safety margins (TSM), denoted by the range of temperatures between $\mathrm{T}_{\text {opt }}$ and $\mathrm{CT}_{\max }$. Performance indices vary, but typically include metabolic rate, aerobic scope, swimming speed, or growth depending on research interests. 


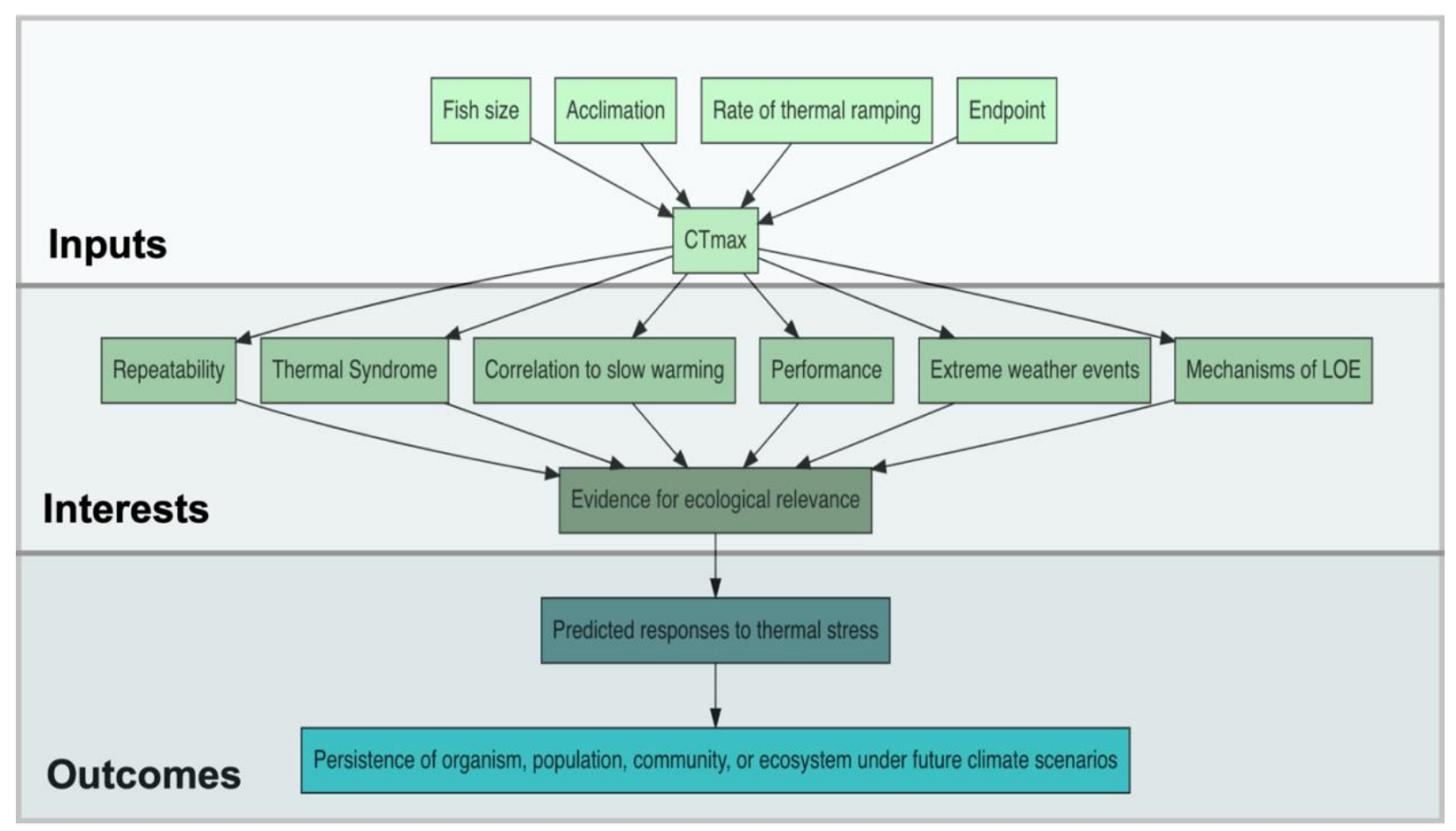

Figure 2-2. CTmax flowchart. A conceptual diagram outlining the links between methodological inputs, research interests, and potential outcomes. 


\section{Boxes}

Box 2-1. On the use of the oxygen-capacity limited thermal tolerance hypothesis to investigate

\section{performance in fishes.}

While there exists a number of conceptual frameworks developed to investigate thermal tolerance in fishes, the oxygen-capacity limited thermal tolerance (OCLTT) hypothesis has received much attention over the years, as it attempts to attribute thermal limits to the decline in aerobic scope at high temperatures (e.g, Fry 1947; Brett 1971; Claireaux and Lefrançois 2007; Pörtner and Knust 2007; Farrell et al. 2008; Pörtner et al. 2008; Pörtner and Farrell 2008; Munday et al. 2009; Nilsson et al. 2009; Pörtner 2010, Pörtner and Peck 2010; Clark et al. 2011; Eliason et al. 2011; Donelson et al. 2012; Munday et al. 2012; Pörtner et al. 2012, Pörtner et al. 2017). Many studies have focused on the use of aerobic scope to develop TPCs in the context of the OCLTT (see Lefevre et al. 2020). However, the validity and utility of this hypothesis has been the subject of much controversy among the ecophysiological community (Jutfelt et al. 2018). Under certain contexts, tissue oxygen limitation has been shown to cause deterioration in whole-animal performance, but there also exists an abundance of evidence that fails to support fundamental assumptions of this hypothesis, hindering the generality of OCLTT mechanisms (see Jutfelt et al. 2018, Lefevre et al. 2021).

Although the particularities of the OCLTT hypothesis are beyond the scope of this paper, it is important to note that using aerobic scope to generate TPCs also has its limitations. It is commonly believed that aerobic capacity is linked to Darwinian fitness (Fry 1971; Pörtner and Farrell 2008), because fish will die if they are unable to escape from predators or unable to forage. However, direct evidence of this relationship is lacking since fish may rarely utilize their full aerobic scope, making it difficult to predict how either short-term or long-term decreases in aerobic capacity would impact fitness in the wild (Lefevre et al. 2021). Moreover, some species have shown vastly different TPCs using aerobic scope; some increase exponentially as temperatures increase, others maintain aerobic scope, and many do not show the typical unimodal curve (Lefevre et al. 2021). As with other performance traits, the shape of aerobic scope performance curves could depend on life stage, geographic location, and biotic or abiotic environmental factors (Lefevre et al. 2021). Furthermore, physiological energetics such as growth or specific dynamic action have been speculated to provide a more relevant way to generate TPCs because they relate more closely to fitness and are uniform across species, although these alternatives still present logistical challenges (Lefevre et al. 2021). 
Box 2-2. Research needs. We identified research priorities by using word cloud software to extract the top 50 words from each of the five considerations discussed in the previous sections. We then generated a subsequent word cloud using these extracted words to narrow down the ten most common terms discussed. This method yielded the following list of words: climate, estimates, stress, conditions, change, acute, rate, time, species, and fitness. We opted to add the term context, as it is central to research in the field of thermal biology. As such, we present the following list of nine themes (acute, rate, and time were grouped) to help focus research aimed at optimizing the use of CTmax in the context of ecology.

\section{Research needs}

\section{Climate}

Understanding how CTmax relates to historical, present, and predicted climate scenarios will provide insight on how individuals, populations, and species will respond to temperature fluctuations and extreme weather events. Identifying patterns in CTmax estimates that coincide with historical extreme weather events, whether observed at local or regional scales, within-populations or across species, will further highlight the relationship between CTmax and survival, a proxy for Darwinian fitness.

2. Estimates

As with any metric of thermal tolerance, there is uncertainty associated with the underlying mechanisms of LOE, which is why they are considered estimates (with some uncertainty) of upper thermal limits. Reducing this uncertainty - either by standardizing protocols or integrating CTmax with functional metrics - will improve accuracy in forecasting responses to warming.

3. Thermal stress

The physiological and biochemical pathways that modulate thermal stress responses at different time scales (resistance, acclimation, adaptation) are not fully understood. Investigating how thermal stress manifests itself across levels of biological organization (cellular to whole-organism) will assist in linking CTmax to performance and fitness.

4. Conditions

Environmental conditions play an immense role in shaping thermal tolerance limits. Conducting CTmax trials in a field-based setting with wild fish can demonstrate more realistic links between this estimate of thermal tolerance and behavioural or physiological responses.

5. Change

Although current evidence suggests that evolutionary rescue might not be possible (Morgan et al. 2019), further understanding the interplay between rates of environmental change and genetic change will be critical in assessing how warming will threaten different species. This is particularly important when considering CTmax estimates, as they can be heavily influenced by rates of thermal ramping.

6. Acute, time, and rate

Future studies should acknowledge that CTmax measures responses to acute thermal stress and emphasize the importance of duration when conducting trials. The duration can physiologically and biochemically impact responses to thermal stress. Efforts should focus on determining the factors that underpin LOE to bridge the gap between acute and chronic thermal tolerance estimates.

7. Species

Fishes are incredibly diverse, and so responses to thermal stress may not be the same across species. As such, developing a systematic way of adjusting CTmax protocols to account for these differences would yield standardized results that could be used in meta-analyses and studies focused on interspecific differences.

8. Fitness

Fitness has been the ultimate focus of past and present studies on upper thermal tolerance, as researchers investigate performance traits such as swimming speed, aerobic scope, metabolic rates, fecundity, and growth. Understanding how these traits manifest themselves under acute thermal challenges will clarify the ecological relevance of CTmax.

9. Context

CTmax methodologies change according to research questions and context. Accounting for factors variables as sex, population, and life stage (among others) is critical when considering species resilience. 


\title{
Chapter 3: Upper thermal tolerance indicated by CTmax fails to predict migration strategy and timing, growth, and predation vulnerability in juvenile brown trout (Salmo trutta)
}

\begin{abstract}
Partial migration is common in a variety of taxa and has important ecological and evolutionary implications, yet the underlying factors that lead to different migratory strategies are not clearly understood. Given the importance of temperature in serving as a cue for migration along, its role in regulating metabolism, growth, reproduction, and survival, we examined how intraspecific variation in critical thermal maxima (i.e., CTmax) influenced migratory strategy (residency vs. migration), the timing of migration, growth, and predation vulnerability in a wild population of partially-anadromous juvenile brown trout (Salmo trutta). Using passive integrated transponder (PIT) telemetry and mark-recapture techniques, we identified individuals that outmigrated to sea, that assumed residency, and that were predated by cormorants several months later. Acute thermal stress induced by conducting CTmax trials did not affect the final fate of assayed fish compared to controls. We found that mass and body condition predicted CTmax and migration timing, but CTmax failed to predict migratory strategy or timing, growth (of resident fish), or predation vulnerability. Although there may be links between mass, thermal tolerance, and migration strategy, the relationship between CTmax and migration remains unclear. The role of upper thermal tolerance in influencing life-history strategies should not be neglected however, as alternative indicators of thermal tolerance could be further explored. The high degree of variation in CTmax estimates warrants additional investigation of how increasingly prevalent high temperature events might drive selection towards thermally-tolerant extremes, which is
\end{abstract}


particularly relevant in a rapidly warming world.

\section{Introduction}

Over the past century, temperature has been identified as one of the most influential factors involved in regulating growth, survival, metabolism, and reproduction in ectotherms (e.g., Fry 1971). As such, there has been much interest in determining optimal temperatures that enhance fitness, in addition to identifying critical thermal limits where performance is impacted (sometimes to the extent where fitness is zero). The critical thermal maximum or CTmax was originally defined by Cowles and Bogert (1944) for ectotherms as "the thermal point at which locomotory activity becomes disorganized and the animal loses its ability to escape from conditions that will promptly lead to its death" (Cowles and Bogert 1944). Though this definition gave rise to much variation among protocols over time, CTmax typically involves acute thermal ramping from acclimation temperatures to an endpoint where either loss of equilibrium (LOE) or the onset of muscle spasms is reached. The relative simplicity of this experimental design has led to a wealth of publications where CTmax is applied in the context of environmental science to identify regulatory guidelines for thermal pollution originating from anthropogenic sources (Lutterschmidt and Hutchison 1997).

However, there has been a shift in the applications of CTmax in the literature over the past few decades as researchers recognize the need to predict individual, population, and specieslevel responses to climate change (e.g. Deutsch et al. 2008; Sandblom et al. 2016; Comte and Olden 2017). Several studies have demonstrated range shifts in response to increases in temperature, indicating the importance of temperature in species distribution (Thomas et al. 2001; Parmesan and Yohe 2003; Brander et al. 2003; Perry et al. 2005; Sunday et al. 2012; Pinsky et al. 2019). CTmax, among other methods, has been used to infer thermal tolerance in 
relation to changes in behaviour and distribution induced by a warming environment in a variety of ectotherms (e.g. Sandblom et al. 2016, Comte and Olden 2017), and may therefore serve as a tool to identify variation in thermal tolerance within populations.

As novel applications of CTmax estimates emerge in recent literature, a number of studies have identified concerns regarding the ecological relevance of such metrics, specifically with regards to the variations in protocols, the rapid rates of experimental temperature increase that are rarely reflective of natural conditions, and the failure of physiological systems, such as the heart, before LOE occurs (Becker and Genoway 1979; Lutterschmidt and Hutchison 1997; Clark et al. 2008; Bates and Morley 2020). Despite these concerns, several recent publications present evidence that validates the use of carefully considered CTmax protocols in an ecological context. In particular, CTmax has been found to be repeatable within individuals over both short (weeks: Morgan et al. 2018; Grinder et al. 2020) and long periods of time (months: O’Donnell et al. 2020). The positive correlation between repeatability and heritability suggests that upper thermal tolerance is a heritable trait, as many studies have previously shown (e.g., Perry et al. 2005, Anttila et al. 2013, Muñoz et al. 2014, Muñoz et al. 2015; Morgan et al. 2018), with the potential to drive evolution under particular conditions. Additionally, Åsheim et al. (2020) recently demonstrated that thermal tolerance to rapid warming rates positively correlates with thermal tolerance evaluated under a more natural, slow warming rate, implying that CTmax values derived using rapid temperature increases provide an ecologically-relevant proxy for upper thermal tolerance. Given the evidence that supports individual repeatability of CTmax, individuals that consistently show higher upper thermal tolerance could perform or behave different that counterparts that display consistently lower upper thermal tolerance. Moreover, the behavioural responses to critical temperatures (LOE or onset of muscle spasms) has been found 
to be consistent throughout a diversity of taxa, suggesting that it is a highly conserved trait (Lutterschmidt and Hutchison 1997). The long history of CTmax, along with recent attempts to validate its use in ecology warrant further applications to continue making advances in the field of thermal biology.

Despite the abundance of CTmax applications, there has been very little focus on how intraspecific variation in upper thermal tolerance estimates contribute to the fitness of individual animals. Understanding how variation in CTmax among individuals contributes to variation in fitness will enable us to predict whether animals are capable of rapid adaptation in response to thermal stress, while simultaneously providing insights into the underlying factors that lead to differences in life-history strategies.

Here, we used CTmax as a proxy for relative upper thermal tolerance in 294 brown trout Salmo trutta, following an adapted version of the protocol described in Morgan et al. (2018), to assess the role of upper thermal tolerance in promoting the fitness of individual fish. We tested whether differences in CTmax would lead to differences in migration strategy or timing, given that stream temperatures are typically warmer than ocean temperatures, and thus individuals with lower CTmax may be driven to migrate, in order to stay within optimal thermal limits. Although stream temperatures in our study system rarely exceed CTmax estimates for brown trout provided in the literature, the fast warming rates correlate with ecologically-relevant slow warming rates ( $\AA$ sheim et al. 2020). Thus, CTmax is a sufficiently sensitive measure to infer differences in thermal stress below sub-lethal temperatures. Once these estimates were obtained, we used Passive Integrated Transponders (PIT) to determine the timing of outmigration towards the sea. After the migration period, mark-recapture techniques were used to re-sample the instream population to assess the proportion of individuals that assumed residency in the natal 
stream. A control treatment with temperatures kept at ambient levels was used to assess whether acute thermal shock influenced migration, survival, and growth.

Brown trout are freshwater salmonids native to various regions of Europe that display partial migration; they can either reside within their natal streams or migrate out to sea. Partial migration is a fascinating phenomenon that represents extreme trade-offs (Chapman et al. 2011a).

Migration is energetically costly and associated with high mortality due to predation, but migrants can exploit a wider range of resources, often leading to similar or better lifetime fitness than residents due to increased size and thus increased reproductive output (Gross 1987; Jonsson and Jonsson 1993; Chapman et al. 2011a). While it is considered one of the most common types of migratory strategy in a variety of taxa, partial migration has critical evolutionary and ecological consequences (Jonsson and Jonsson 1993; Nilsson et al. 2008; Grayson and Wilbur 2009; Hebblewhite and Merrill 2009; Chapman et al. 2011b). Despite this importance, the factors underpinning the decision to migrate or assume residency remain unclear. The decision is thought to be linked to both genetics and environmental factors (Ferguson et al. 2019, Lemopoulos et al. 2019), suggesting that temperature and genetic traits that underlie the mechanisms for coping with thermal stressors may impact these decisions. Moreover, individual condition, gill Na/K-ATPase activity, and energetic status have also been shown to play a role in the decision and timing of migration (Aarestrup et al. 2000; Nielsen et al. 2004; Nielsen et al. 2006; Wysujack et al. 2009; Boel et al. 2014; Peiman et al. 2017). Given the documented importance of temperature in regulating migration timing and success in salmonids (e.g. Jonsson 1991; Aarestrup et al. 2002; Sloat and Reeves 2014), we tested the hypothesis that individual CTmax would predict the decision to either migrate or assume residency, and also be linked to the timing of migration, survival, and growth, 
Phenotypic variation has been shown to account for variation in migration timing in wild searun brown trout (Bohlin et al. 1996), though mechanisms underlying these differences are still poorly understood. Temperature is a key environmental factor that is known to modulate physiological functions such as growth, metabolism, reproductive success, and migration (Ficke et al. 2007; Jonsson and Jonsson 2009; Sloat and Reeves 2014). Factors that affect physiological functions (both directly and indirectly), such as food availability, temperature, and increased water discharge (among others), may affect the decision to migrate or assume residency (Aarestrup et al. 2002; Archer et al. 2020). For example, high temperatures may lead to higher metabolic rates, such that fish require more food to sustain adequate growth rates and meet higher metabolic demands. If there is insufficient food available to sustain these demands, fish could have lower body condition and may migrate to sea in search of additional resources (Armstrong et al. 2010). The interactions between temperature, food availability, metabolism and condition have been shown to influence the decision to migrate or assume residency (Økland et al. 1993; Boel et al. 2014; Sloat and Reeves 2014).

Although there may be an interplay between temperature, metabolism, and food availability, our focus here was to understand whether individual differences in CTmax can predict migration strategy and timing, given that warmer stream temperatures may induce stress in fish with lower CTmax. There is already evidence demonstrating intraspecific differences in thermal tolerance among different populations of salmonids that undergo migrations of variable difficulty (Eliason et al. 2011). Thus, thermal tolerance may be linked to various aspects of migration; it may drive migration in individuals with lower thermal tolerance if stream temperatures rise above optimal temperatures. By using CTmax as a relative measure of upper thermal tolerance, we explored the role of upper thermal tolerance in mediating life-history strategies. In turn, this can highlight the 
importance of phenotypic variation in the distribution of animals in a progressively warming climate.

\section{Materials and Methods}

\section{Study site}

Wild brown trout were obtained from Gudsø stream, which is located in the southern region of Jutland, Denmark (Fig. 1). The stream is home to an abundant population of partially migrant brown trout (Birnie-Gauvin et al. 2017; Peiman et al. 2017). The stream flows for $6 \mathrm{~km}$ and is 1 to 4 meters wide, allowing for efficient mark-recapture electrofishing. The Gudsø stream reaches Kolding Fjord, which exits into the southern end of the Kattegat Sea. Two PIT stations are located roughly 500 and $600 \mathrm{~m}$ from the outlet of the stream. Migrant brown trout in this population often spend between 0.5 and 2 years in freshwater, after which they migrate to sea, where they typically spend 1-2 years (Birnie-Gauvin and Aarestrup 2019). Though this population is home to migrants and residents, the majority consists of migratory individuals (10:1 ratio), as is the trend for most brown trout populations with access to sea (e.g. Jonsson 1985, Midwood et al. 2015, Birnie-Gauvin et al. 2017). Over the past two decades or so, summer temperatures ranged from 9.7 to $19.5^{\circ} \mathrm{C}$, averaging around $13.8^{\circ} \mathrm{C}$. Although brown trout are known to tolerate these temperatures quite well (Forseth et al. 2009), individual variation in thermal tolerance may result in some conspecifics being more sensitive to fluctuations in temperatures, and may account for differences in behaviour, condition, and migratory phenotypes. 


\section{Capturing, measuring, and tagging}

From the $11^{\text {th }}$ to the $15^{\text {th }}$ of February 2020, a total of 614 brown trout ranging from 11.0 to $22.7 \mathrm{~cm}$ were captured from a stretch of the stream approximately one kilometer upstream of the PIT stations (Fig.1) using single-pass electrofishing gear (Scubla ELT 60 II G, running at $300 \mathrm{~V})$. The use of electrofishing as a method to collect wild trout for this study was justified by a previous study that found that electrical shocks did not have an effect on CTmax results (Carline and Machung 2001). Fish were netted immediately and placed into a 60-L container of freshly oxygenated stream water, where water was changed frequently. Additional oxygen was provided via air stones to ensure appropriate holding conditions were met. Once fish were captured, they were anesthetized in a $0.3 \mathrm{~g} \mathrm{l}^{-1}$ benzocaine solution, measured for total length $(1 \mathrm{~mm})$ and wet mass (0.1 g). Fulton's condition factor (K, equation 1, Ricker 1975) was calculated for each fish using total length and weight measurements.

$$
\text { Condition Factor }(K)=\frac{\operatorname{mass}(\mathrm{g})}{\text { length }(\mathrm{cm})^{3}} \times 100
$$

The fish were then tagged using a $23 \mathrm{~mm}$ PIT tag (Texas Instruments, RI-TRP-RRHP, $134 \mathrm{~Hz}, 0.6 \mathrm{~g}$ mass in air, Plano, Texas, USA). PIT tags have been shown to be effective tools to monitor and identify particular individuals while posing virtually no negative impact on the fish, provided they have the appropriate body size to accommodate the tag (Gibbons and Andrews 2004). Previous studies have also shown good retention rates of PIT tags $(97 \%$ in a similar

species and system, Larsen et al. 2013), providing us with a good method for monitoring fish long-term. 


\section{Experimental Design}

The experimental tanks consisted of two 60-L opaque containers filled with approximately $17.5 \mathrm{~L}$ of well-oxygenated stream water: one for a control group and one for the CTmax group that experienced thermal ramping. Each container was supplied with a pump to circulate water at low flow rates (EHEIM air400 10W), air stones to keep oxygen levels $>70 \%$ saturation, and a metal mesh separating the fish from the equipment. In the CTmax tank, two heaters (EHEIM ThermoControl 250W and EHEIM JÄGER 300W) were included and set to the highest setting to achieve a rate of temperature increase of $0.3^{\circ} \mathrm{C} \mathrm{min}^{-1}\left(18^{\circ} \mathrm{C} \mathrm{h}^{-1}\right)$, as suggested in previous studies (see Morgan et al. 2018; Lutterschmidt and Hutchison 1997; Becker and Genoway 1979). Water temperatures were monitored using a recently factory-calibrated thermometer (Traceable 4052 Long-Stem Thermometer S/N: 191869383, $\pm 1 \square \mathrm{C}$ accuracy), in addition to temperature data loggers (HOBO TidbiT v2 Temperature Data Logger) that were set to record temperatures every second for the duration of the trials. Oxygen was monitored using a recently calibrated handheld dissolved oxygen meter (OxyGuard Handy Polaris).

Prior to each trial, the tanks were filled with fresh stream water such that the experiment began at the temperatures at which the fish were acclimated to in their natural environment, which ranged from 4.5 to $5.7^{\circ} \mathrm{C}$ during the course of the experiments. While we did not measure whether the fish were fully acclimated (i.e., maintaining a new stable physiological state), we opted to use the term 'acclimation' to refer to wild fish that had a prolonged exposure to natural fluctuations in temperature. Then, 20 fish were randomly selected from the holding containers for each group (control or CTmax) and were placed into the experimental containers, at which point the heaters in the CTmax container were turned on and thermal ramping began. Temperatures were kept at ambient levels in the control container. The fish were monitored from 
a distance to observe changes in behaviour, swimming performance, onset of muscles spasms that led to LOE, which we defined as the endpoint in accordance with several studies (e.g. Baroudy and Elliott 1994; Becker and Genoway 1979; Galbreath et al. 2006; O’Donnell et al. 2020). The fish were removed from the experimental container when they lost their ability to maintain dorso-ventral orientation, which clearly marks LOE. Once removed from the experimental tank, the brown trout were placed into a recovery container supplied with stream water at ambient temperature. Most fish recovered and displayed normal behaviour in less than five minutes, indicating that the thermal challenge did not cause significant trauma. Only five fish were unable to recover from the temperature treatment (98.41\% survival). Once the fish recovered, they were released in the stream near the site of capture. Multiple trials were conducted each day, until the designated sample size of approximately 300 fish per treatment was reached.

\section{CTmax}

CTmax was used to quantify relative differences in thermal tolerances among 294 individual brown trout exposed to thermal ramping rates of approximately $18^{\circ} \mathrm{C} \mathrm{h}^{-1}$ (average of $0.32 \pm 0.02^{\circ} \mathrm{C} \mathrm{min}^{-1}$ ) in accordance with previous studies (Becker and Genoway 1979; Lutterschmidt and Hutchison 1997; Morgan et al. 2018). Fluctuations in air and river temperatures both prior and during the experimental trials resulted in slight deviations from the desired rate of thermal ramping $\left(0.3^{\circ} \mathrm{C} \mathrm{min}-1\right)$. This caveat is addressed by accounting for the time exposed to temperatures above acclimation while reporting critical thermal maximum values. Although maximum water temperature in which fish experience LOE is most frequently used as an endpoint for CTmax values (Lutterschmidt and Hutchison 1997; Morgan et al. 2018), a recent study found that using cumulative degree minutes provided a more accurate measure 
since fish with different start temperatures may experience these changes at relatively similar temperatures but quite some time apart (O’Donnell et al. 2020). Incorporating time to LOE also accounts for imperfect heating rates within experimental trials due to mechanical or electrical malfunctions (Galbreath et al. 2004; O'Donnell et al. 2020). For the purposes of this study, it is more appropriate to use cumulative degree minutes rather than temperature readings since fish were acclimated to stream temperatures immediately prior to conducting CTmax trials. Stream temperatures varied temporally with each trial (by a maximum of $0.7^{\circ} \mathrm{C}$ ) as water temperature is highly correlated with air temperature. Fish in trials with lower starting temperatures will experience higher temperatures for a longer period of time before reaching LOE, potentially subjected to a higher degree of thermal stress. Rates of thermal ramping were also slightly different across trials, which further warrants the use of a standardized metric to compare fish from different trials. Additionally, Galbreath et al. (2004) state that mortality due to thermal stress is an additive process, meaning that the time involved in coping with higher than usual temperatures should be accounted for while measuring relative tolerance between fish within an experiment (Kilgour and McCauley 1986; Galbreath et al. 2004). In this case, using cumulative degree minutes $(\mathrm{CDM})$ as a proxy for $\mathrm{CTmax}$ provides a more sensitive measure than raw temperature $\left({ }^{\circ} \mathrm{C}\right)$ for comparing thermal tolerance between individual fish across multiple trials.

\section{Migration}

Two PIT stations were set to continuously detect the passage of tagged fish as they migrated from the stream to the fjord. The two PIT stations were approximately $100 \mathrm{~m}$ apart, each station consisting of two full stream covering antennaes spaced $5 \mathrm{~m}$ apart. The efficiency of the upstream most PIT station was estimated to be approximately $91.2 \%$, based on fish that were detected at both PIT stations as well as fish that were only detected at the downstream most PIT 
station (see Zydlewski et al. 2006). For the purpose of this study, we assume that the efficiency of the downstream most PIT station is similar to the upstream one, though the specific efficiency of the downstream station cannot be calculated (Zydlewski et al. 2006). The stream was resampled on the $8^{\text {th }}$ of June 2020 , which is considered to be after the typical spring migration period for juvenile brown trout in this system. Fish that were detected at the upstream station and the downstream station in that order were defined as 'migrants', while fish that were simply recaptured within the stream in June were defined as 'residents'. For the purpose of this study, we assume that the fish that passed both PIT stations, the upstream and downstream stations, in that order, migrated. While there is a $500 \mathrm{~m}$ stretch of stream between the downstream PIT station and the sea, previous samplings found no previously tagged fish in that stretch as it is not suitable habitat for brown trout (personal communications, K. Birnie-Gauvin). Fish with no detections that were not recaptured within the river were categorized to be of 'unknown strategy'. Fish that were detected at only one of the PIT stations and were not recaptured in the stream were also considered as fish of 'unknown strategy', as directionality of movement could not be confirmed.

\section{Growth}

Specific growth rate (SGR, equation 2) was calculated for each fish that was recaptured over a 4 months-time interval (i.e., all residents), from the day of capture to the day of recapture.

$$
S G R=\frac{\ln (\text { final mass })-\ln (\text { initial mass })}{\# \text { days post sampling }} \times 100
$$




\section{Survival and Predation}

Survival and predation can be inferred by PIT station detection efficiency, electrofishing recapture efficiency, and detection of PIT tags that were found at cormorant colonies near Gudsø stream. High detection efficiency $(91.2 \%)$ and high recapture rates achieved in narrow streams $(>$ $90 \%$, see Midwood et al. 2015) provide sufficient grounds to assume mortality of fish that were not recaptured nor detected at PIT stations. Fish that were detected at cormorant colonies were classified as 'Predated', regardless of their migratory strategy, providing a larger sample size to enhance the detection of potential links with CTmax.

\section{Statistical analyses}

Data were first examined for homogeneity of variance, collinearity, and outliers. Among the 614 fish that were sampled and used in the experiments, 5 CTmax fish ( $0.8 \%$ of all fish) did not recover from the experiment and were thus removed from the analyses. Preliminary analysis showed autocorrelation between length and mass, therefore only mass was considered in the models. Model explanatory variables were also inspected to ensure normality. Mass was logtransformed twice to meet underlying assumptions of the models.

Welch's t-tests were used to investigate whether there were differences in initial body mass, length, and condition across control and CTmax fish. ANOVAs were also used to evaluate whether body metrics and CDM differed across final status (resident, migrant, unknown fate, predated). These analyses were also performed with raw temperature values at which LOE occurred for further validation of the observed trends with CDM. A Pearson's chi-square analysis was used to determine whether the proportion of fish with different final status differed between the control and CTmax trials. 
Cumulative degree minutes (CDM) was used as a proxy for CTmax, which adjusted upper thermal tolerances indexes to include effects of different acclimations temperatures and rates of thermal ramping across trials. Linear regression models were used to evaluate whether body metrics (mass and condition factor) affected CTmax. The final model included both mass and initial condition factor as explanatory variables.

Generalized linear models (GLMs) were used to investigate potential relationships between CDM and life history choices (reside or migrate, and the corresponding timing of migration). We followed a step-by-step approach of model simplification from a full model that included all explanatory variables (CDM, mass, and condition) and all two-way interactions, using Akaike's information criterion (AIC). We also ensured models were distinguishable from one another by comparing $\triangle \mathrm{AICs}$, where a difference $<2$ was considered to be more or less equivalent (Bolker 2008). We then proceeded to check for overdispersion based on the $\chi^{2}$ approximation of residual deviance and continued with model validation to ensure all assumptions were met.

The decision to migrate was modelled as a binomial response, where migrants were classified as 'successes' (1) and residents as 'failures' (0), with CDM, mass and condition as explanatory variables. A sample size of 151 fish was used in this analysis (i.e., only CTmax fish with known fates). The final model included only mass as explanatory variable with log-log link function. Similarly, a GLM was used to explore whether CDM, mass or condition affected the timing of migration. Using a Poisson distribution resulted in an overdispersion leading to the use of a negative binomial distribution with log-link function in the analysis. The final model included mass and initial condition factor as explanatory variables. A bimodal distribution of outmigration timing led us to group migrants in two groups, early (0-30 days post-sampling) and 
late (31+ days post-sampling). A non-parametric t-test was used to compare differences in initial mass of early and late migrants, which further validated the use of mass as a predictor variable in the best model. A t-test was used to explore differences in CTmax between early and late migrants.

GLMs were also used to explore whether CTmax was related to growth in residents (as only residents were recaptured). CTmax estimates and specific growth rates (SGR, \% growth per day since sampling) were available for only 8 resident fish, so only one explanatory variable could be examined per model. A series of GLMs were conducted with mass, initial condition, and CDM as explanatory variables to investigate relationships with SGR. When comparing AIC values to the null model, the model including mass provided the best fit.

Finally, a series of GLMs with binomial distribution and logit link function were used to investigate whether predation by cormorants was affected by CDM, mass, and condition. Models included only one explanatory variable at a time due to low sample size $(N=26)$. The best model was the null model, suggesting that none of the included explanatory variables had an effect on predation.

All statistical analyses were performed using R version 3.6.1 using lattice, ggplot2, and MASS R packages (R Core Team 2016; Sarkar 2008; Wickham 2016; Venables and Ripley 2002, respectively).

\section{Results}

A total of 614 juvenile brown trout were initially captured, tagged, and sampled. Of those fish, 320 were exposed to control conditions while 294 were exposed to acute thermal ramping at a rate of $0.32 \pm 0.02^{\circ} \mathrm{C} \mathrm{min}^{-1}$. Upon resampling, a total of 22 brown trout ranging from 13.2 to $24.7 \mathrm{~cm}$ were recaptured. Given that the peak of the typical migration season occurs in the 
spring, we assume that these fish have assumed residency in their natal stream. PIT data confirmed a total of 288 migrants, while 304 fish were neither detected nor recaptured and consequently labelled as 'unknown strategy'. It is possible that fish of 'unknown strategy' may not have been recaptured during resampling, though electrofishing in this stream is typically high ( $>90 \%$, see Midwood et al. 2015, Birnie-Gauvin 2017). These fish are likely dead due to predation by either cormorants, otters, or seals, or due to other natural causes like competition or disease. High rates of mortality during the winter could also account for a large proportion of fish of 'unknown strategy' (Midwood et al. 2015). Among the 614 fish that were tagged, 68 (11.07\%) were detected in nearby cormorant colonies, 26 of which had CTmax measurements and 42 of which were control fish. Note that these 68 predated fish included fish classified as migrants, residents and fish of 'unknown strategy'.

\section{Acute thermal shock}

To assess whether migratory strategy or final fate were influenced by acute thermal stress induced by the experimental approach used to measure CTmax, we used a control group in which fish were exposed to similar handling conditions but lacked acute thermal ramping. A total of 145 of the $288(45 \%)$ migratory fish and 14 of the $22(64 \%)$ residents were from control treatments. The experimental approach did not affect the fate or migratory strategy of fish $\left(\chi^{2}=\right.$ $0.445, P>0.05$, Table 3-1).

Initial condition was found to be marginally higher in the CTmax group $(t=-2.18, P=$ 0.030). The slightly lower condition factor observed among fish in the control group could indicate preparation for a parr-smolt transformation in several control fish (see Boel et al. 2014), leading to higher migration propensity within the control group. However, this marginal difference in condition factor does not appear to have affected fish final status as evidenced by 
similar proportions of migrants, residents, and fish of unknown strategy in both CTmax and control groups (Table 3-1).

\section{CTmax}

We found that mass was positively correlated to $\operatorname{CDM}(t=4.96, P<0.001)$, while initial condition was negatively correlated to $\operatorname{CDM}(t=-2.468, P=0.014)$. The best model $\left(F_{2,291}=\right.$ 15.73, $\left.P<0.0001, R^{2}=0.091\right)$ included both mass $(t=4.96, P<0.001)$ and initial condition $(t=-$ 2.47, $P=0.014$ ) as explanatory variables (Fig. 3-2).

Of the 294 fish that experienced thermal ramping, 143 (48.6\%) migrated, 8 (5.6\%) resided within the stream, and 143 (48.6\%) were of 'unknown strategy'. Twenty-six fish were found to have been predated upon, representing $8.8 \%$ of the treatment group. Mean CDM was similar across all groups (ANOVA, all $\mathrm{P}>0.05$, Table 3-2). These results were further confirmed using raw temperature values where LOE occurred rather than CDM, though CTmax was found to be similar across fish with different final statuses.

\section{Migratory strategy}

Mass was not associated with migratory strategy $(Z=-1.588, P>0.05)$. CDM was not included as an explanatory variable in the best model, suggesting that that CTmax does not play a role in the decision to migrate or not.

\section{Timing of migration}

We observed two peaks in the timing of migration: one occurring 0-30 days postsampling ( end of February), the other 31+ days post-sampling (April-May). We did not find any indication that CTmax differed among early or late migrants $(t=1.500, P=0.137)$. We found that the number of days spent in the river before migration was negatively related to mass 
$(Z=-2.685, P=0.007$, Fig. $3-3 \mathrm{~A})$ but not to condition $(Z=1.689, P=0.091)$, suggesting that larger fish migrated earlier. This is further supported by a direct comparison of mass between early ( $\leq 30$ days post-sampling, $N=54$, mean $=36.8$, s.d. $=19.6)$ and late $(\geq 31$ days postsampling, $N=88$, mean $=27.4$, s.d. $=19.6$ ) migratory groups, where we found early migrants to have significantly greater mass on average than late migrants $(P=0.008$, Fig. 3-3B).

\section{Growth}

Of the fish that were exposed to acute thermal stress and later recaptured in the stream (i.e., residents, $N=8$ ), mass was positively correlated to SGR $(t=5.443, P=0.002)$, such that residents that were larger at the time of tagging grew faster. Similar trends were observed among control fish that assumed residency $(N=14, t=11.05, P<0.001)$, suggesting that acute thermal stress did not influence growth rates. We found no difference $(t=-0.36, P=0.723)$ in SGR between control fish $(x=405.18, N=14)$ and CTmax fish $(x=412.48, N=8)$, indicating that acute thermal shock did not affect growth rates in resident fish. Since these models are limited by a low sample size, caution should be taken when interpreting this result.

\section{Predation}

We found no significant differences in rate of predation $\left(\chi^{2}=2.43, P=0.119\right)$ in fish from different groups (i.e. control or CTmax). Thus, acute thermal shock did not increase the probability of being predated. Mean CDM for predated fish was found to be 637.5 , and did not differ from the mean $\mathrm{CDM}$ of fish that were not predated (average $=646, t=0.55, \mathrm{df}=32, P=$ 0.587). Neither mass, condition nor CDM predicted likelihood of predation by cormorants (GLMs, all $P>0.05$ ). 


\section{Discussion}

The phenotypic differences between migratory and resident brown trout are not well understood. This study found a range of CTmax values within a population of semi-anadromous brown trout (Table 3-2); this variation could be beneficial under different thermal conditions. Given the recent evidence supporting the ecological relevance of CTmax, we investigated whether these differences were related to the decision and timing of migration. We speculated that fish with low CTmax values may be more likely to migrate, as stream temperatures tend to be warmer than sea temperatures, and thus may act as a thermal stressor. We further speculated that differences in CTmax values may affect migration timing, survival and growth, given that temperature is viewed as a primary factor affecting metabolism, growth, reproduction and survival (Fry 1971), but we found no indication that intraspecific variation in CTmax predicted migration strategy, timing, growth, or predation vulnerability. Regardless, the role of upper thermal tolerance in mediating migration propensity and timing could be further explored by considering alternative indicators of thermal tolerance such as the expression of heat shock proteins (Fangue et al. 2006). Similarly, morphological and physiological differences related to oxygen transport amongst individuals within a population could also provide insight on the role of thermal tolerance in regulating life-history strategies in salmonids (Eliason et al. 2011).

While several studies have found relationships between mass, condition (or both) and the decision to migrate or assume residency, the relationship between condition and mass with thermal tolerance indicated by CTmax appears to be rather subtle. We found that CTmax correlated positively with mass and negatively with condition. Although CTmax did not predict the decision to migrate or the timing of migration, our results suggest that high body mass and low body condition are associated with higher CTmax in juvenile brown trout. However, the 
relationship between body size and condition with CTmax has yet to be validated in wild fish populations. Previous studies report positive correlations (e.g. Bard and Kieffer 2019, Zhang and Kieffer 2014), while others report no correlations or negative correlations with mass (e.g. O’Donnell et al. 2020, Morgan et al. 2018). Discrepancies in CTmax experimental protocols among studies may mask relationships between fish size and CTmax, where rapid heating rates may result in lags between environmental and core body temperatures in larger fish (Zhang and Kieffer 2014; Becker and Genoway 1979; Lutterschmidt and Hutchison 1997).

Compared to residents, migratory individuals have been found to either be smaller (Archer et al. 2020; Peiman et al. 2017; Morinville and Rasmussen 2003; Theriault and Dodson 2003) or larger (Bohlin et al. 1994; Olsson et al. 2006, Acolas et al. 2012), and with lower body condition than residents (Archer et al. 2020; Peiman et al. 2017; Wysujack et al. 2009; Boel et al. 2014). The present study did not find any significant associations between body metrics and migratory tactics, though relatively few residents were recaptured and low sample sizes may have hindered our ability to detect patterns.

In addition to its correlation to CTmax, mass was also correlated to migration timing, a finding which is supported by previous studies (e.g., Birnie-Gauvin et al. 2017; Metcalfe et al. 1990; Bohlin et al. 1996). We found that larger individuals tended to migrate earlier and higher thermal tolerance. Since mass is known to positively correlate with metabolic rate (Økland et al. 1993; Thorpe et al. 1998), the metabolic demands of larger individuals are likely higher, and thus these individuals require more resources to sustain metabolic demands and high growth rates. By entering the sea earlier, larger individuals gain greater feeding opportunities earlier, thus enabling them to meet their metabolic demands. This idea also supports the size threshold hypothesis that larger individuals migrate earlier (Økland et al. 1993; Bohlin et al. 1996). These results 
demonstrate that individual variation in migration timing (which is a function of temperature and flow) is a result of phenotypic variation (Bohlin et al. 1996). While we did not find any correlations between variation in CTmax and timing of migration, phenotypic variation in thermal tolerance may still account for variation in the timing of migration but CTmax may not be the most appropriate method for detecting this link. Considering the importance of temperature in triggering migration, future studies should focus on the use of alternative metrics of thermal tolerance such as measuring maximum metabolic rates and aerobic scope under warm (sub-lethal) temperatures.

The intrinsic correlation between mass and metabolism provides a foundation to speculate about the role of thermal tolerance in mediating migration timing. The idea that larger individuals have higher CTmax and migrate earlier due to higher metabolic demands could indicate that traits involved in regulating thermal tolerance are likely to be acting on metabolic rates as well. Since metabolism has been hypothesized to influence the timing of migration in brown trout (Økland et al. 1993), it is also conceivable that a link exists between upper thermal tolerance and timing of migration. Predicted temperature fluctuations associated with climate change may simultaneously alter metabolism and thermal tolerance, and lead to shifts in migration timing (Whitney et al. 2016). Changes in the timing of migration could have important and wide-ranging effects on ecosystems, and result in misalignments between resource availability and arrival of migratory individuals.

Although CTmax did not predict the timing of migration in this study, the relationship could be masked by average thermal conditions. Here, maximum stream temperatures $\left(19.5^{\circ} \mathrm{C}\right)$ remain approximately $2{ }^{\circ} \mathrm{C}$ below the minimum CTmax measured $\left(21.68^{\circ} \mathrm{C}\right)$, suggesting that fish do not currently experience sufficient selective pressure to drive a difference in CTmax between 
residents and migrants. With progressively warmer stream temperatures predicted in the near future (IPCC 2014), thermal conditions might reveal a stronger relationship between CTmax and migration strategy and timing. A recent review found that fish perceive sudden exposure to high temperature as a stressor, inducing high levels of cortisol and catecholamines (Alfonso et al. 2020). In turn, high levels of cortisol have been shown to correlate with earlier migration in salmonids (Birnie-Gauvin et al. 2019). Increasingly prevalent extreme weather events, such as heat waves, could promote outmigration of fish with low CTmax if warmer stream temperatures induce stress responses. Understanding the role of individual thermal tolerance indicated by CTmax in relation to life history strategies under future climate scenarios is an interesting avenue for future research.

\section{Limitations}

It is important to address the limitations associated with the uncertainty regarding the ultimate fate of individuals. Though PIT station detection efficiency was high $(91.2 \%)$, some individuals may have migrated without detection, just as some individuals that assumed residency may not have been recaptured within the stream during resampling or detected at cormorant colonies. For the purpose of this study, we assumed that fish from both the control or treatment groups had equal chances of being undetected, predated, or recaptured. Moreover, even larger sample sizes would be required in future studies to facilitate comparisons between migrants and residents in populations where migratory phenotypes typically exceed residents. Low sample sizes of resident trout in this study makes it particularly difficult to detect significant interactions, especially those relevant to CTmax. However, mean CDM for residents exceeded that of other final statuses by approximately 40 units, while others groups are fairly similar. 
Repeating this study with larger sample sizes would enhance statistical power and potentially alter the findings of this study.

It is also worth noting that CTmax measurements were somewhat low compared to other studies on brown trout (e.g. Carline and Machung 2001, Galbreath et al. 2004). This may be due to the effect of photoperiod or seasonal changes on thermal tolerance, as well as differences in experimental protocols (e.g., acclimation and rates of thermal ramping). There is an abundance of literature stating that longer days (i.e. during the summer) lead to higher upper thermal tolerance (Lutterschmidt and Hutchison 1997). In this study, wild trout were captured in the winter, thus exposed to short daylengths and low temperatures, and were likely acclimated to cooler temperatures than if they had been captured in the summer. In the wild, fish are exposed to constant fluctuations in air and water temperatures, and may explain differences observed between wild fish and those kept under controlled laboratory conditions prior to trials. For these reasons, the CTmax values obtained here should not be directly compared to other studies assessing thermal tolerance in brown trout, unless similar conditions and methodologies were used. Finally, we assumed that CTmax estimates obtained in this study were repeatable, given the recent evidence found in several species over a range of time scales. For the purpose of this study, we expected fish with relatively high CTmax to consistently display higher upper thermal tolerance and vice-versa. However, if CTmax is not repeatable in wild juvenile brown trout, correlations to acute upper thermal tolerance found in this study may also not be repeatable.

\section{Conclusions}

To our knowledge, this is the first study that attempts to link individual variation in CTmax to migratory tactics in a partially migrant population of wild fish. After estimating CTmax in 294 wild juvenile brown trout, we found no evidence that this metric of upper thermal 
tolerance affected migratory propensity, though the generality of our result is somewhat hindered by a low sample size of resident fish. We found that larger fish migrated earlier, consistent with previous findings. We also found that larger fish, and fish in better condition, had higher CTmax values, but CTmax did not predict probability of migration, growth, or vulnerability to predation. Although CTmax did not predict migration strategy, further investigating the relationships between metabolism, mass, and thermal tolerance using maximum metabolic rate and aerobic scope measurements could provide insight into the potential role of upper thermal tolerance in determining life-history strategies. We speculate that CTmax may play more important roles in the determination of life-history strategies, growth or predation in populations that are exposed to more frequent extreme heat events. Future research should focus on understanding how these extreme weather events impact thermal tolerance in wild populations and how these changes subsequently influence migration propensity, timing, growth, and predation. While CTmax remains a common tool in the field of thermal biology, its role in mediating life-history strategies remains unclear. Given the urge to manage, conserve, and protect biodiversity in a rapidly warming world, such tools are important to understand how temperature may impact population dynamics in the wild.

\section{Ethical standards}

All procedures and experimental work was conducted in accordance with the [institutional protocols] (2017-15-0201-01164). 


\section{Tables}

Table 3-1. Summary of tagged fish. Mean initial length (cm), mass (g), and condition (K) for individually tagged Salmo trutta for each treatment group ( \pm s.d.). The proportion of fish either migrating, residing or of unknown strategy within each group is shown as a percentage.

Subscript letters indicate significant differences between treatments. Note that predated fish include residents, migrants, and unknown fish.

\begin{tabular}{lll}
\hline \multirow{2}{*}{ Metric } & Treatment & \\
\cline { 2 - 3 } & Control & CTmax \\
\hline Sample size & 320 & 294 \\
Mass $(\mathrm{g})$ & $31.50 \pm 19.20$ & $32.86 \pm 19.68$ \\
Length $(\mathrm{cm})$ & $14.1 \pm 2.79$ & $14.3 \pm 2.8$ \\
Condition $(\mathrm{K})$ & $1.01 \pm 0.08_{\mathrm{A}}$ & $1.02 \pm 0.08 \mathrm{~B}$ \\
\% Migrants & $45.31(145)$ & $48.64(143)$ \\
\% Residents & $4.38(14)$ & $2.72(8)$ \\
\% Unknown & $50.31(161)$ & $48.64(143)$ \\
\% Predated & $13.13(42)$ & $8.84(26)$ \\
\hline
\end{tabular}

Table 3-2. CTmax by final status. Total number $(\mathrm{N})$, mean \pm s.d., minimum, and maximum cumulative temperature (cumulative degree minutes) and temperature $\left({ }^{\circ} \mathrm{C}\right.$ ) at loss of equilibrium for individually tagged Salmo trutta exposed to thermal ramping based on status as of June $8^{\text {th }}$, 2020. Note that Predation category includes fish categorized as migrants, residents, and unknown.

\begin{tabular}{lllllll}
\hline \multirow{2}{*}{ Final status (N) } & \multicolumn{2}{l}{ Cumulative Temperature (CDM) } & \multicolumn{2}{l}{ Temperature $\left({ }^{\circ} \mathrm{C}\right)$} & \\
& Mean & Min. & Max. & Mean & Min. & Max. \\
\hline Total (294) & $645.25 \pm 85.88$ & 395.65 & 943.33 & $25.63 \pm 1.49$ & 21.68 & 30.08 \\
Migrants (143) & $646.12 \pm 78.37$ & 422.78 & 932.36 & $25.65 \pm 1.48$ & 21.73 & 30.08 \\
Residents (8) & $685.47 \pm 85.67$ & 617.1 & 870.98 & $26.52 \pm 1.15$ & 25.51 & 28.7 \\
Unknown (143) & $642.12 \pm 92.80$ & 395.65 & 943.33 & $25.56 \pm 1.51$ & 21.68 & 29.52 \\
Predation (26) & $645.25 \pm 85.88$ & 395.66 & 943.33 & $25.63 \pm 1.49$ & 21.68 & 30.08 \\
\hline
\end{tabular}


Table 3-3. Final statuses metric summary. Total number $(\mathrm{N})$, mean \pm s.d., and range of body metrics obtained during initial sampling of control and CTmax Salmo trutta in February 2020 according to final statuses. Note that Predation category includes fish categorized as migrants, residents, and unknown.

\begin{tabular}{llll}
\hline Final status $(\mathrm{N})$ & Length $(\mathrm{cm})$ & Mass $(\mathrm{g})$ & Condition $(\mathrm{K})$ \\
\hline Total $(614)$ & $14.2 \pm 2.7$ & $32.13 \pm 19.43$ & $1.017 \pm 0.084$ \\
& $(11.0-22.7)$ & $(11.0-115.6)$ & $(0.663-1.360)$ \\
Migrants (288) & $14.1 \pm 2.6$ & $31.20 \pm 17.8$ & $1.012 \pm 0.082$ \\
& $(11.0-21.5)$ & $(12-115.4)$ & $(0.828-1.360)$ \\
Residents (22) & $15.3 \pm 3.1$ & $40.28 \pm 25.3$ & $1.015 \pm 0.061$ \\
& $(11.0-22.7)$ & $(14.4-115.6)$ & $(0.927-1.113)$ \\
Unknown (304) & $14.2 \pm 2.8$ & $32.42 \pm 20.4$ & $1.021 \pm 0.087$ \\
& $(11.0-21.7)$ & $(11.0-107.6)$ & $(0.663-1.331)$ \\
Predation (68) & $14.5 \pm 2.8$ & $33.97 \pm 19.39$ & $1.008 \pm 0.072$ \\
& $(11.0-20.6)$ & $(12.0-87.0)$ & $(0.784-1.229)$ \\
\hline
\end{tabular}




\section{Figures}

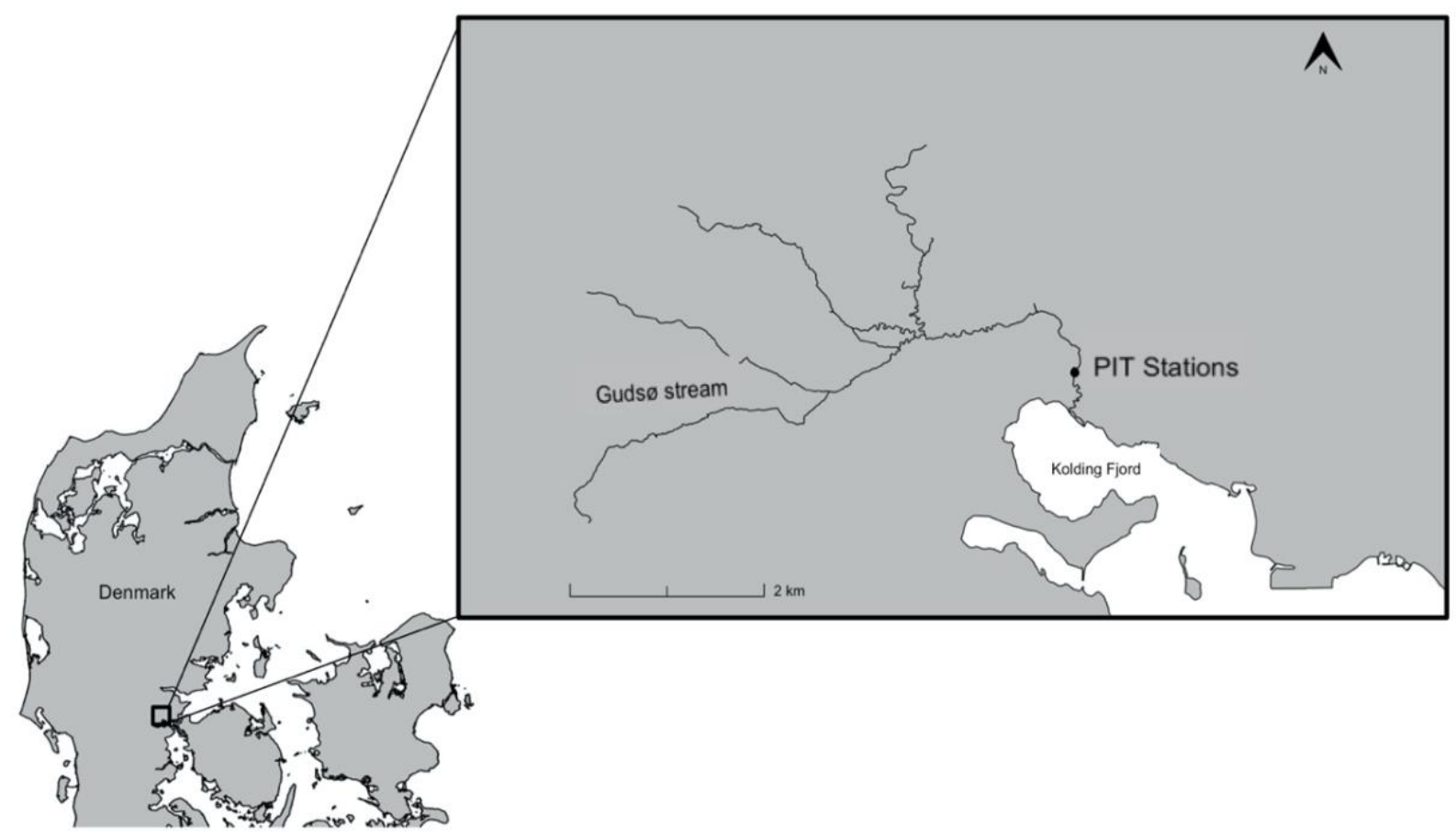

Figure 3-2. Gudsø study site map. Location of study site, Gudsø stream, Jutland, Denmark. PIT stations are indicated by black circle. Traced lines represent areas where the stream runs and the outflow location of the stream into the Kolding Fjord. 

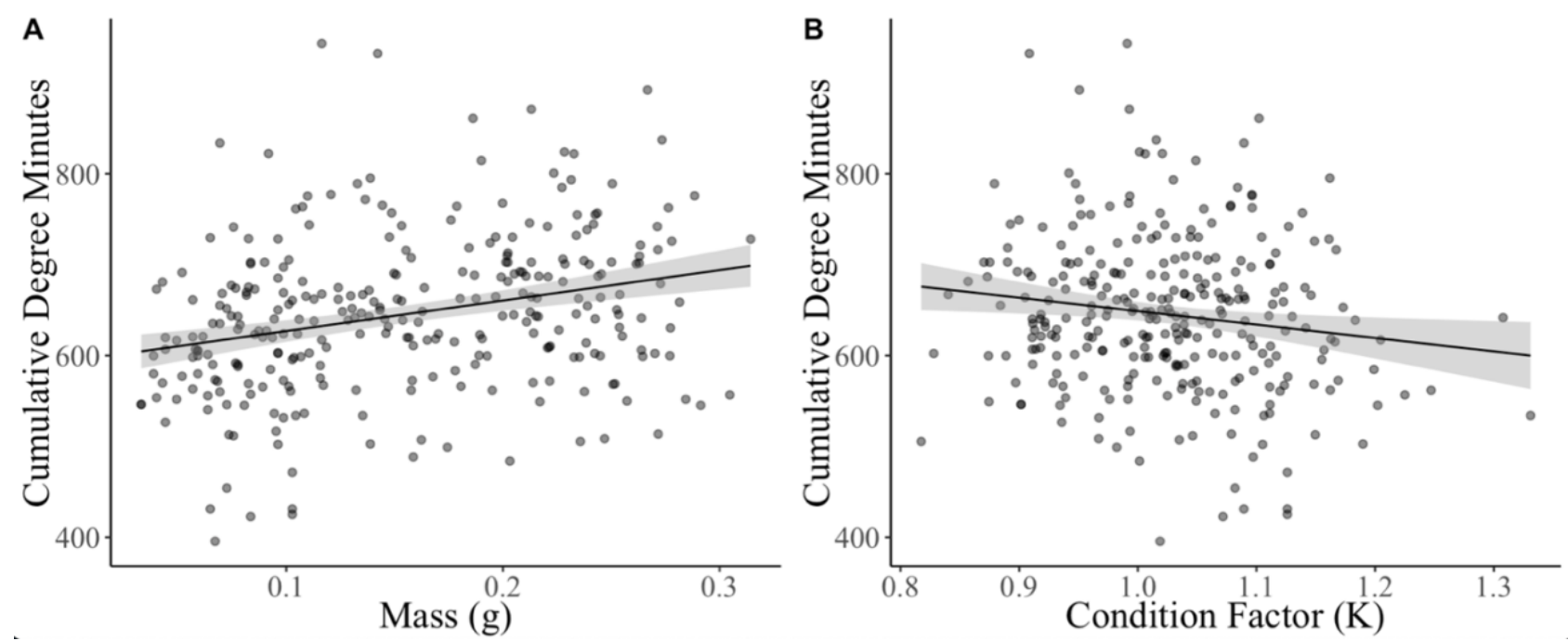

Figure 3-2. CTmax and mass/condition. Modelled Cumulative Degree Minutes (CDM) as a function of (A) mass (log-log transformed, with initial condition held at mean values) and (B) initial condition factor (K, with mass held at mean values) in juvenile Salmo trutta. (A) suggests a positive relationship between mass and CDM while (B) suggests a negative relationship between condition factor and CDM. Shaded areas represent $95 \%$ confidence intervals. 

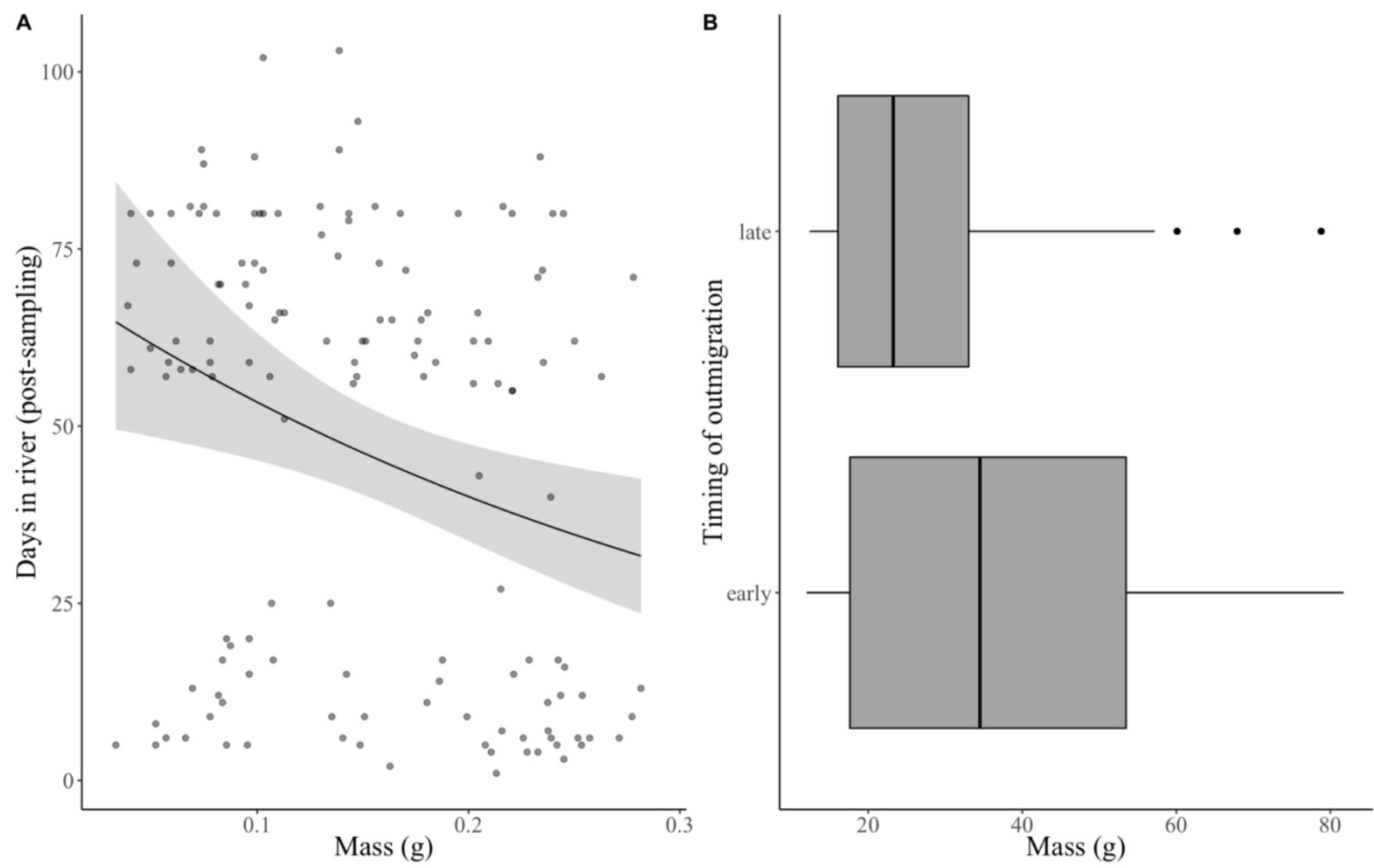

Figure 3-3. Migration timing. (A) Modelled probability of timing of migration (days spent in river after sampling) as a function of mass (log-log transformed, with initial condition held at mean values) in juvenile Salmo trutta illustrating a significant negative relationship between mass and migration timing $(Z=-2.685, P=0.007)$. Shaded areas represent $95 \%$ confidence intervals. (B) Distribution of initial mass (g) demonstrating significantly greater mass $(P=$ 0.008) of early migrants ( 0 -30 days post-sampling, $N=54)$ compared to late migrants $(31+$ days post-sampling, $N=88$ ). 


\section{Chapter 4: General Discussion and Conclusion}

Although thermal tolerance can be measured in several different ways depending on study objectives, CTmax remains one of the most logistically feasible techniques since it requires very little resources and time. As with any alternative method, there are constraints and procedures that should be followed to preserve the integrity of this experimental design and ensure that acute thermal limits are measured. In Chapter 2, I reviewed the evidence supporting the ecological relevance of CTmax and suggested an array of directions for future research to further outline how this technique can be used in field of fish ecology. Some evidence for ecological relevance includes 1) the fact that LOE is commonly observed in a variety of taxa, 2) that CTmax was found to be repeatable in laboratory-reared fishes, implying that there is a genetic component, 3) that there is a link between CTmax estimates and species' geographic distribution, and 4) that acute rates of thermal ramping do exist in the wild, and tolerance to these correlates with tolerance to slower rates of ramping.

In Chapter 3, I exemplified how CTmax can be used in field-based studies to examine ecologically-relevant phenomena, such as migration propensity and timing, growth and predation vulnerability in a population of semi-anadromous juvenile brown trout. While there was no evidence to suggest direct links between CTmax and migration propensity or timing, these findings indicate potential relationships between CTmax, mass, and metabolism. In this case, using CTmax in addition to measuring aerobic scope and maximum metabolic rate to predict differences in these fitness-related proxies would provide further insight on the role of thermal tolerance in mediating these outcomes. Moreover, using CTmax according to suggested guidelines and disclosing all aspects of the experimental trial (including acclimation rates and duration, rate of thermal ramping, and endpoint) in combination with alternative metrics can 
generate a better understanding of the ecological relevance of this technique. This empirical study attempted to link CTmax to performance traits such as growth and ability to avoid predators in wild fishes. Although we did not find a significant relationship, we found evidence that may suggest correlations between mass, metabolism, and thermal tolerance. As such, in addition to demonstrating how CTmax can be applied to investigate intraspecific differences in thermal tolerance in a wild population of fish, we also provided fundamental information that could lead to better understanding of the underlying mechanisms of CTmax.

There is sufficient evidence to warrant the integration of CTmax methods in the study of thermal tolerance. However, relying solely on this approach for predicting responses to warming will likely ignore some important aspects of thermal tolerance and only generate a vague representation of future responses. Despite the frequent use of CTmax estimates for making climate change-related predictions, it is also critical to understand that CTmax can be used to identify organisms that might be more vulnerable to thermal stress induced by infrastructure planning such as electricity generation or stormwater management (e.g. Turko et al. 2020). When constrained by time and resources, CTmax has been shown to be an effective tool to gain some insight on populations, communities, or ecosystems that may be most vulnerable to thermal stress. Finally, while thermal tolerance encompasses a diverse array of complex terms, it is equally important to emphasize that these limits are heavily influenced by time and space. As such, characterizing absolute thermal limits for individuals or species presents an immense challenge. Instead, attempts made to advance the current state of knowledge by synthesizing relevant information and applying findings to investigate ecological phenomena may be of greatest value. 
Among the suggestions I provided for future research in Chapters 2 and 3, linking CTmax to functional performance metrics such as growth, swimming ability, or life-history strategies will be particularly important in terms of using CTmax to predict responses to thermal stress. This could be done by directly measuring these performance traits across an acute rate of thermal ramping with acclimated fish. This could also be investigated indirectly, by using more functional indices of thermal tolerance (e.g., decline in aerobic scope, cardiac performance, change in metabolic rate) to changes in growth, swimming ability, or other performance traits. After obtaining correlations between these two variables, evaluating how CTmax correlates with the alternative index of thermal tolerance would then provide insight on performance traits, and in turn, the ecological relevance of CTmax. An equally important area of future research could focus on the possibility of individuals demonstrating a 'thermal type'. Understanding whether individuals with repeatably low or high CTmax consistently show different physiological or behavioural responses to various challenges will further highlight the role of acute upper thermal limits in ecology. Investigating the possibility of a thermal syndrome is relatively new, but promising area of research that could refine our current predictive models. Finally, using CTmax to investigate thermal tolerance and responses to thermal stress at different levels of biological organization, from molecules to ecosystems, could explain some of the complex interactions that have been limiting advances in the field of thermal biology. 


\section{Literature Cited}

Aarestrup K., Nielsen, C. and Madsen S.S. (2000). Relationship between gill Na+, K+-ATPase activity and downstream movement in domesticated and first-generation offspring of wild anadromous brown trout (Salmo trutta). Canadian Journal of Fisheries and Aquatic Sciences, 57, 2086-2095.

Aarestrup, K., Nielsen, C. and Koed, A. (2002). Net ground speed of downstream migrating radio-tagged Atlantic salmon (Salmo salar L.) and brown trout (Salmo trutta L.) smolts in relation to environmental factors. Hydrobiologia, 483, 95-102.

Acolas, M.L., Labonne, J. and Bagliniere J. L. (2012). The role of body size versus growth on the decision to migrate: a case study with Salmo trutta. Naturwissenchaften 99, 11-21.

Alfonso, S., Gesto M. and Sadoul, B. (2020). Temperature increase and its effects on fish stress physiology in the context of global warming. Journal of fish biology, 98, 1496-1508.

Angilletta, M.J. (2009). Thermal acclimation. In Thermal Adaptation. Oxford University Press, New York, pp. 126-156.

Anttila K., Dhillon, R.S. , Boulding, E.G., Farrell, A.P., Glebe, B.D., Elliott, J.A., Wolters, R.W. and Schulte, P.M. (2013). Variation in temperature tolerance among families of Atlantic salmon (Salmo salar) is associated with hypoxia tolerance, ventricle size and myoglobin level. Journal of Experimental Biology, 216, 1183-1190.

Archer, L.C., Hutton, S.A., Harman, L., McCormick, S.D., O’Grady, M.N., Kerry, J.P, Poole, W.R, Gargan, P., McGinnity, P. and Reed, T.E. (2020). Food and temperature stressors have opposing effects in determine flexible migration decisions in brown trout. Global Change Biology, 26, 2878-2896.

Armstrong, J.B., Schindler, D.E., Omori, K.L., Ruff, C.P. and Quinn, T.P. (2010). Thermal heterogeneity mediates the effects of pulsed subsidies across a landscape. Ecology, 91: 14451454.

Åsheim E., Andreassen, A.H., Morgan, R. and Jutfelt. F. (2020). Rapid-warming tolerance correlates with tolerance to slow warming but not growth at non-optimal temperatures in zebrafish. Journal of Experimental Biology, 223, jeb229195.

Bard, B. and Kieffer, J.D. (2019). The effects of repeat acute thermal stress on the critical thermal maximum (CTmax) and physiology of juvenile shortnose sturgeon (Acipenser brevirostrum). Canadian Journal of Zoology, 97, 576-572.

Bard, B. (2020). Chronic Social Stress Impairs the Thermal Tolerance of Rainbow Trout (Oncorhynchus Mykiss) (Doctoral dissertation, Université d'Ottawa/University of Ottawa). 
Baroudy, E. and Elliott, J.M. (1994). The critical thermal limits for juvenile Arctic charr Salvelinus alpinus. Journal of Fish Biology, 45,1041-1053.

Bates, A.E. and Morley, S.A. (2020). Interpreting empirical estimates of experimentally derived physiological and biological thermal limits in ectotherms. Canadian Journal of Zoology, 98, 237-244.

Becker, C.D. and Genoway, R.G. (1979). Evaluation of the critical thermal maximum for determining thermal tolerance of freshwater fish. Environmental Biology of Fish, 4, 245-256.

Beitinger, T.L., Bennett, W.A., and McCauley, R.W. (2000). Temperature tolerances of North American freshwater fishes exposed to dynamic changes in temperature. Environmental biology of fishes, 58, 237-275.

Beitinger, T.L., Bennett, W.A. (2000) Quantification of the role of acclimation temperature in temperature tolerance of fishes. Environmental Biology of Fishes 58, 277-288.

Bell, A.M., Hankison, S.J. and Laskowski, K.L. (2009). The repeatability of behaviour: a metaanalysis. Animal Behaviour, 77, 771-783.

Bell, G. 2013. Evolutionary rescue and the limits of adaptation. Philosophical Transactions of the Royal Society B: Biological Sciences, 368, 20120080-20120080.

Birnie-Gauvin, K. and Aarestrup, K. (2019). A call for a paradigm shift: Assumed_ to_ be premature migrants actually yield good returns. Ecology of Freshwater Fish, 28, 62-68.

Birnie-Gauvin, K., Flávio, H. Kristensen, M.L., Walton-Rabideau, S., Cooke, S.J., Willmore, W.G., Koed, A. and Aarestrup, K. (2019). Cortisol predicts migration timing and success in both Atlantic salmon and sea trout kelts. Science Reports, 9, 1-9.

Birnie-Gauvin, K., Peiman, K.S., Larsen, M.H., Baktoft, H., Aarestrup, K., Willmore, W.G. and Cooke, S.J. (2017). Oxidative stress and partial migration in brown trout (Salmo trutta). Canadian Journal of Zoology, 95, 829-835.

Blasco, F.R., Esbaugh, A.J., Killen, S.S., Rantin, F.T., Taylor, E.W. and McKenzie, D.J. (2020). Using aerobic exercise to evaluate sub-lethal tolerance of acute warming in fishes. Journal of Experimental Biology, 223, jeb218602

Boake, C.R.B. (1989). Repeatability: its role in evolutionary studies of mating behavior. Evolutionary Ecology, 3, 173-182.

Boel, M., Aarestrup, K., Baktoft, H., Larsen, T., Madsen, S., Malte, H., Skov, C., Svendsen, J.C. and Koed, A. (2014). The Physiological Basis of the Migration Continuum in Brown Trout (Salmo trutta). Physiological and Biochemical Zoology, 87, 334-345. 
Bohlin, T., Dellefors, C. and Faremo, U. (1994). Probability of first sexual maturation of male parr in wild sea-run brown trout (Salmo trutta) depends on condition factor $1 \mathrm{yr}$ in advance. Canadian Journal of Fisheries and Aquatic Sciences, 51, 1920-1926.

Bohlin, T., Dellefors, C. and Faremo, U. (1996). Date of smolt migration depends on body-size but not age in wild sea-run brown trout. Journal of Fish Biology, 49, 157-164.

Bolker, B. 2008. Ecological Models and Data in R. Princeton University Press.

Brander, K., Blom, G., Borges, M.F., Erzini, K., Henderson, G., MacKenzie, B.R., Mendes, H. Ribeiro, J., Santos, A.M.P. and Toresen, R. (2003). Changes in fish distribution in the eastern North Atlantic: Are we seeing a coherent response to changing temperature? ICES Marine Science Symposia 219, 261-270.

Brett, J.R. (1971). Energetic response of salmon to temperature. A study of some thermal relations in the physiology and fresh-water ecology of sockeye salmon (Oncorhynchus nerka). American. Zoology, 11, 99-113.

Carline, R.F. and Machung, J.F. (2001). Critical Thermal Maxima of Wild and Domestic Strains of Trout. Transactions of the American Fisheries Society, 130, 1211-1216.

Carter, W.A. (1887). Temperature in relation to fish. Nature, 36, 213-214.

Chapman, B.B., Bronmark, C., Nilsson, J-A. and Hansson, L-A. (2011a). The ecology and evolution of partial migration. Oikos, 120, 1764-1775.

Chapman, B.B., Hulten, K., Blomqvist, D.R., Hansson, L-A, Nilsson, J-A., Broderson, J., Nilsson, P.A., Skov, C. and Bronmark, C. 2011b. To boldly go: individual difference in boldness influence migratory tendency. Ecology Letters, 14, 871-876.

Chevin, L.M. and Lande, R. (2010). When do adaptive plasticity and genetic evolution prevent extinction of a density-regulated population?. Evolution: International Journal of Organic Evolution, 64, 1143-1150.

Chown, S.L., Jumbam, K.R., Sørensen, J.G., and Terblanche, J.S. (2009). Phenotypic variance, plasticity and heritability estimates of critical thermal limits depend on methodological context. Functional Ecology, 23, 133-140.

Christensen, E.A., Norin, T., Tabak, I., van Deurs, M. and Behrens, J.W. (2021). Effects of temperature on physiological performance and behavioral thermoregulation in an invasive fish, the round goby. Journal of Experimental Biology, 224, p.jeb237669.

Chung, K.S. (2001). Critical thermal maxima and acclimation rate of the tropical guppy Poecilla reticulata. Hydrobiologia, 462, 253-257. 
Clark, T.D., Sandblom, E., Cox, G.K., Hinch, S.G. and Farrell. A.P. (2008). Circulatory limits to oxygen supply during an acute temperature increase in the Chinook salmon (Oncorhynchus tshawytscha). American Journal of Physiology-Regulatory, Integrative and Comparative Physiology, 295, R1631- R1639.

Claireaux, G. and Lefrançois, C. (2007). Linking environmental variability and fish performance: integration through the concept of scope for activity. Philosophical Transactions of the Royal Society B, 362, 2031-2041.

Clark, M.S., Fraser, P.P., Peck, L.S. (2008). Antarctic marine molluscs do have an HSP70 heat shock response. Cell Stress Chaperones, 13, 39-49.

Clark, T.D., Jeffries, K.M., Hinch, S.G. and Farrell, A.P. (2011). Exceptional aerobic scope and cardiovascular performance of pink salmon (Oncorhynchus gorbuscha) may underlie resilience in a warming climate. Journal of Experimental Biology, 214, 3074- 3081.

Clark, T.D., Roche, D.G., Binning, S.A., Speers-Roesch, B. and Sundin, J. (2017). Maximum thermal limits of coral reef damselfishes are size dependent and resilient to near-future ocean acidification. Journal of Experimental Biology, 220, 3519-3526.

Claireaux, G., Théron, M., Prineau, M., Dussauze, M., Merlin, F. X., and Le Floch, S. (2013). Effects of oil exposure and dispersant use upon environmental adaptation performance and fitness in the European sea bass, Dicentrarchus labrax. Aquatic Toxicology, 130, 160-170.

Comte, L. and Olden, J.D. (2017). Climatic vulnerability of the world's freshwater and marine fishes. Nature Climate Change, 7, 718-722.

Coutant, C.C. and Brook, A.J. (1970). Biological aspects of thermal pollution I. Entrainment and discharge canal effects. Critical Reviews in Environmental Science and Technology, 1, 341-381.

Cowles, R.B. and Bogert, C.M. (1944). A preliminary study of the thermal requirements of desert reptiles. Bulletin of the AMNH; v. 83, article 5. The Quarterly Review of Biology, 20, 170170 .

Cox, D.K. (1974). Effects of three heating rates on the critical thermal maximum of bluegill. In: J. W. Gibbons and R. R. Sharitz (ed.) Thermal Ecology, CONF-730505, Nat. Tech. Inf. Serv. Springfield, V. A. pp. 158-163.

Davy, J. (1863). Some observations on the vitality of fishes, as tested by increase of temperature. In: Report of the 32nd Meeting of the British Association for the Advancement of Science Notices. John Murray, London, p. 125.

Desforges, J.E., Birnie-Gauvin, K., Aarestrup, K. and Cooke, S.J. (2021). Upper thermal tolerance indicated by CTmax fails to predict migration strategy and timing, growth, and predation vulnerability in juvenile brown trout Salmo trutta. Physiological and Biochemical Zoology, 94, 215-227. 
Deutsch, C.A., Tewksbury, J.J., Huey, R.B., Sheldon, K.S., Ghalambor, C.K., Haak, D.C. and Martin, P.R. (2008). Impacts of climate warming on terrestrial ectotherms across latitude. Proceedings of the National Academy of Sciences of the United States of America, 105, 66686672.

Dillon, M.E., Wang, G., Huey, R.B. (2010). Global metabolic impacts of recent climate warming. Nature, 467, $704-706$.

Dingemanse, N.J., Kazem, A.J., Réale, D., and Wright, J. (2010). Behavioural reaction norms: animal personality meets individual plasticity. Trends in ecology \& evolution, 25, 81-89.

Dohm, M. R. (2002). Repeatability estimates do not always set an upper limit to heritability. Functional Ecology, 16, 273-280.

Dochtermann, N.A., Schwab, T. and Sih, A. (2015). The contribution of additive genetic variation to personality variation: heritability of personality. Proceedings of the Royal Society B: Biological Sciences, 282, 20142201.

Donelson, J.M., Munday, P.L., McCormick, M.I. and Pitcher, C.R. (2012). Rapid transgenerational acclimation of a tropical reef fish to climate change. Nature Climate Change, $2,30-32$.

Dowd, W.W., King, F.A., Denny, M.W. (2015). Thermal variation, thermal extremes and the physiological performance of individuals. Journal of Experimental Biology, 218, 1956 - 1967.

Ekström, A., Brijs, J., Clark, T. D., Gräns, A., Jutfelt, F., and Sandblom, E. (2016). Cardiac oxygen limitation during an acute thermal challenge in the European perch: effects of chronic environmental warming and experimental hyperoxia. American Journal of PhysiologyRegulatory, Integrative and Comparative Physiology, 311, R440-R449.

Eliason, E.J., Clark, T.D., Hague, M.J., Hanson, L.M., Gallagher, Z.S., Jeffries, K.M., Gale, M.K., Patterson, D.A., Hinch, S.G. and Farrell, A.P. (2011). Differences in Thermal Tolerance Among Sockeye Salmon Populations. Science, 332, 109-112.

Elliott J.M. and Elliott J.A. (1995) The effect of the rate of temperature increase on the critical thermal maximum for parr of Atlantic salmon and brown trout. Journal of Fish Biology, 47, 917 919.

Ern, R., Norin, T., Gamperl, A.K. and Esbaugh, A.J. (2016). Oxygen dependence of upper thermal limits in fishes. Journal of Experimental Biology, 219, 3376-3383.

Ern, R., Johansen, J.L., Rummer, J.L. and Esbaugh, A.J. (2017). Effects of hypoxia and ocean acidification on the upper thermal niche boundaries of coral reef fishes. Biological Letters, 13, 20170135. 
Falconer, D.S. and Mackay, T.F.C. (1996). Introduction to Quantitative Genetics (fourth ed.). Prentice Hall, Harlow, U.K.

Fangue, N.A., Hofmeister, M. and Schulte, P.M. (2006). Intraspecific variation in thermal tolerance and heat shock protein gene expression in common killifish, Fundulus heteroclitus. Journal of Experimental Biology, 209, 2859-2872

Farrell, A.P., Hinch, S.G., Cooke, S.J., Patterson, D.A., Crossin, G.T., Lapointe, M. and Mathes, M.T. (2008). Pacific salmon in hot water: applying aerobic scope models and biotelemetry to predict the success of spawning migrations. Physiological and Biochemical. Zoology, 81, 697708.

Ferguson, A., Reed, T.E., Cross, T.F., McGinnity, P. and Prodöhl, P.A. (2019). Anadromy, potamodromy and residency in brown trout Salmo trutta: the role of genes and the environment. Journal of Fish Biology 95, 692-718.

Ficke, A., Myrick, C.A. and Hansen, L.J. (2007). Potential impacts of global climate change on freshwater fisheries. Reviews in Fish Biology and Fisheries, 17, 581-613.

Finnegan, S., Heim, N.A., Peters, S.E. and Fischer, W.W. (2012). Climate change and the selective signature of the Late Ordovician mass extinction. Proceedings of the National Academy of Sciences of the United States of America, 109, 6829-6834.

Forseth, T., Barlaup, B.T., Finstad, B., Fiske, P., Gjøsæter, H., Falkegård, M., Hindar, A., Mo, T.A., Rickardsen, A.H., Thorstad, E.B., Vøllestad, L.A. and Wennevik, V. (2017). The major threats to Atlantic salmon in Norway. ICES Journal of Marine Science, 74, 1496-1513.

Fry, F.E.J. (1947). Effects of the environment on animal activity. Publications of the Ontario Fisheries Research Laboratory, 68, 1-52.

Fry, F.E.J. (1971). The effect of environmental factors on the physiology of fish. Fish physiology 1,98 .

Galbreath, P.F., Adams, N.D. and Martin, T.H. (2004). Influence on heating rate on measurement of time to thermal maximum in trout. Aquaculture 241, 587-599.

Galbreath, P.F., Adams, N.A., Sherrill, L.W. and Martin, T.H. (2006). Thermal tolerance of diploid versus triploid rainbow trout and brook trout assessed by time to chronic lethal maximum. Environmental Biology of Fishes 75, 183-193.

Gibbons, J.W. and Andrews, K.M. (2004). PIT Tagging : Simple Technology at Its Best. BioScience, 54, 447-454.

Gomez Isaza, D.F., Cramp, R.L., Smullen, R., Glencross, B.D. and Franklin, C.E. (2019) Coping with climatic extremes: dietary fat content decreased the thermal resilience of barramundi (Lates calcarifer). Comparative Biochemistry and Physiology Part A: Molecular \& Integrative Physiology, 230, 64-70. 
Goulet, C. T., Thompson, M.B. and Chapple, D.G. (2017b). Repeatability and correlation of physiological traits: do ectotherms have a 'thermal type'? Ecology and Evolution, 7, 710-719.

Goulet, C.T., Thompson, M.B., Michelangeli, M., Wong, B.B. and Chapple, D.G. (2017a). Thermal physiology: a new dimension of the pace-of-life syndrome. Journal of Animal Ecology, $86,1269-1280$.

Grayson, K.L. and Wilbur, H.M. (2009). Sex- and context-dependent migration in a pongbreeding amphibian. Ecology 90, 306-311.

Gross, M.R. 1987. Evolutionary of diadromy in fishes. American Fisheries Society Symposium 1, 14-25.

Gunderson, A.R. and Stillman, J.H. (2015). Plasticity in thermal tolerance has limited potential to buffer ectotherms from global warming. Proceedings of the Royal Society London B, 282, 20150401.

Hebblewhite, M. and Merrill, E.H. (2009). Trade-offs between predation risk and forage differ between migration strategies in a migratory ungulate. Ecology, 90, 3445-3454.

Holland, W.E., Smith, M.H., Gibbons, J.W. and Brown, D.H. (1974). Thermal Tolerances of Fish from a Reservoir Receiving Heated Effluent from a Nuclear Reactor. Physiological Zoology, 47, 110-118.

Hutchison, V.H. (1961). Critical thermal maximum in salamanders. Physiological Zoology, 43, 92-125.

Hutchison, V.H. (1976). Factors influencing thermal tolerances of individual organisms. ERDA (Energy Res. Dev. Adm.) Symp. Ser. CONF750425. pp. 10-26.

Huey, R.B., Berrigan, D. (2001) Temperature, demography, and ectotherm fitness. American Naturalist, 158, 204-210.

Huey, R.B., Kearney, M.R., Krockenberger, A., Holtum, J.A.M., Jess, M., Williams, S.E. (2012) Predicting organismal vulnerability to climate warming: roles of behaviour, physiology and adaptation. Philosophical Transactions of the Royal Society of London B Biological Sciences, 367, 1665-1679.

Illing, B., Downie, A.T., Beghin, M., and Rummer, J.L. (2020). Critical thermal maxima of early life stages of three tropical fishes: Effects of rearing temperature and experimental heating rate. Journal of Thermal Biology, 90, 102582.

IPCC. (2014). R. K. Pachauri and L. A. Meyer, Climate Change 2014: Synthesis report. contribution of working groups I, II and III to the fifth assessment report of the intergovernmental panel on climate change. Geneva, Switzerland: Core Writing Team. 
IPCC, 2021: Summary for Policymakers. In: Climate Change 2021: The Physical Science Basis. Contribution of Working Group I to the Sixth Assessment Report of the Intergovernmental Panel on Climate Change [Masson-Delmotte, V., P. Zhai, A. Pirani, S. L. Connors, C. Péan, S. Berger, N. Caud, Y. Chen, L. Goldfarb, M. I. Gomis, M. Huang, K. Leitzell, E. Lonnoy, J.B.R. Matthews, T. K. Maycock, T. Waterfield, O. Yelekçi, R. Yu and B. Zhou (eds.)]. Cambridge University Press. In Press.

Isaza, D.F.G., Cramp, R.L., Smullen, R., Glencross, B.D. and Franklin, C.E. (2019). Coping with climatic extremes: dietary fat content decreased the thermal resilience of barramundi (Lates calcarifer). Comparative Biochemistry and Physiology Part A: Molecular \& Integrative Physiology, 230, 64-70.

Jeffries, K. M., Connon, R. E., Davis, B. E., Komoroske, L. M., Britton, M. T., Sommer, T., ..., and Fangue, N.A. (2016). Effects of high temperatures on threatened estuarine fishes during periods of extreme drought. Journal of Experimental Biology, 219, 1705-1716.

Jeffries, K.M., Fangue, N.A., and Connon, R.E. (2018). Multiple sub-lethal thresholds for cellular responses to thermal stressors in an estuarine fish. Comparative Biochemistry and Physiology Part A: Molecular \& Integrative Physiology, 225, 33-45.

Jeffries, K.M., Hinch, S.G., Sierocinski, T., Pavlidis, P. and Miller, K.M. (2014). Transcriptomic responses to high water temperature in two species of $\mathrm{P}$ acific salmon. Evolutionary Applications, 7, 286-300.

Jonsson, B. (1985). Life history patterns of freshwater resident and sea-run migrant brown trout in Norway. Transactions of the American Fisheries Society, 114, 182-194.

Jonsson, B. and Jonsson, N. (1993). Partial migration: niche shift versus sexual maturation in fishes. Reviews in Fish Biology and Fisheries, 3, 348-365.

Jonsson, N. (1991). Influence of water flow, water temperature and light on fish migration in rivers. Nordic Journal Freswater Research, 66, 20-35

Jonsson, B. and Jonsson, N. (2009). A review of the likely effects of climate change on anadromous Atlantic salmon Salmo salar and brown trout Salmo trutta, with particular reference to water temperature and flow. Journal of Fish Biology, 75, 2381-2447.

Jutfelt, F., Roche, D.G., Clark, T.D., Norin, T., Binning, S.A., Speers-Roesch, B., Amcoff, M., Morgan, R., Andreassen, A.H. and Sundin, J. (2019). Brain cooling marginally increases acute upper thermal tolerance in Atlantic cod. Journal of Experimental Biology, 222, p.jeb208249.

Jutfelt, F., Norin, T., Ern, R., Overgaard, J., Wang, T., McKenzie, D.J., Lefevre, S., Nilsson, G.E., Metcalfe, N.B., Hickey, A.J. and Brijs, J. (2018). Oxygen-and capacity-limited thermal tolerance: blurring ecology and physiology. Journal of Experimental Biology, 221, p.jeb169615. 
Kilgour, M. and McCauley, R.W. (1986). Reconciling the two methods of measuring upper lethal temperatures in fishes. Environmental Biology of Fishes, 17, 281-290.

Killen, S.S., Adriaenssens, B., Marras, S., Claireaux, G. and Cooke, S. J. (2016). Context dependency of trait repeatability and its relevance for management and conservation of fish populations. Conservation Physiology, 4(1).

Kingsolver, J.G. and Woods, H.A. (2016). Beyond thermal performance curves: modeling timedependent effects of thermal stress on ectotherm growth rates. American Naturalist, 187, 283294.

Krebs, R.A. and Loeschcke, V. (1994) Costs and benefits of activation of the heat-shock response in Drosophila melanogaster. Functional Ecology, 8, 730.

Kumar, N., Minhas, P.S., Ambasankar, K., Krishnani, K.K., \& Rana, R.S. (2014). Dietary lecithin potentiates thermal tolerance and cellular stress protection of milk fish (Chanos Chanos) reared under low dose endosulfan-induced stress. Journal of thermal biology, 46, 40-46.

Larsen, M.H., Thorn, A.N., Skov, C. and Aarestrup, K. (2013). Effects of passive integrated transponder tags on survival and growth of juvenile Atlantic salmon Salmo salar. Animal Biotelemetry, $1: 19-25$.

Larsson, S. (2005). Thermal preference of Arctic charr, Salvelinus alpinus, and Brown trout, Salmo trutta - implications for their niche segregation. Environmental Biology of Fishes, 73, 89-96.

LeBlanc, S., Middleton, S., Gilmour, K.M. and Currie, S. (2011). Chronic social stress impairs thermal tolerance in the rainbow trout (Oncorhynchus mykiss). Journal of Experimental Biology, 214, 1721-1731.

Lefevre, S., Wang, T. and McKenzie, D.J. (2021). The role of mechanistic physiology in investigating impacts of global warming on fishes. Journal of Experimental Biology, 224(Suppl 1).

Lemopoulos, A., Uusi-Heikkilä, S., Hyvärinen, P., Alioravainen, N., Prokkola, J.M., Elvidge, C.K., Vasemägi, A. and Vainikka, A. (2019). Association mapping based on a common-garden migration experiment reveals candidate genes for migration tendency in brown trout. G3, 9, 2887-2896.

Lutterschmidt, W.I. and Hutchison, V.H. (1997). The critical thermal maximum: history and critique. Canadian Journal of Zoology 75, 1561-1574.

Lydy, M.J. and Wissing, T.E. (1988). Effect of sublethal concentrations of copper on the critical thermal maxima (CTMax) of the fantail (Etheostoma flabellare) and johnny (E. nigrum) darters. Aquatic toxicology, 12, 311-321. 
MacMillan, H.A. (2019). Dissecting cause from consequence: a systematic approach to thermal limits. Journal of Experimental Biology, 222(4).

Madeira, D., Narciso, L., Diniz, M.S. and Vinagre, C. (2014). Synergy of environmental variables alters the thermal window and heat shock response: An experimental test with the crab Pachygrapsus marmoratus. Marine environmental research, 98, pp.21-28.

McArley, T. J., Hickey, A. J. and Herbert, N.A. (2017). Chronic warm exposure impairs growth performance and reduces thermal safety margins in the common triplefin fish (Forsterygion lapillum). Journal of Experimental Biology, 220, 3527-3535.

McKenzie, D.J., Zhang, Y., Eliason, E.J., Schulte, P.M., Claireaux, G., Blasco, F.R., ... \& Farrell, A. P. (2020). Intraspecific variation in tolerance of warming in fishes. Journal of Fish Biology, 98, 1536-1555.

Morgan, R., Finnøen, M.H., Jutfelt, F. (2018). CT $\max$ is repeatable and doesn't reduce growth in zebrafish. Science reports, 8, 7099.

Morgan, R., Finnøen, M.H., Jensen, H., Pélabon, C. and Jutfelt, F. (2020). Low potential for evolutionary rescue from climate change in a tropical fish. Proceedings of the National Academy of Sciences, 117, 33365-33372.

Morley, S.A., Peck, L.S., Sunday, J.M., Heiser, S. and Bates, A.E. (2019). Physiological acclimation and persistence of ectothermic species under extreme heat events. Global Ecology and Biogeography, 28, 1018-1037.

Munday, P.L., Crawley, N.E. and Nilsson, G. E. (2009). Interacting effects of elevated temperature and ocean acidification on the aerobic performance of coral reef fishes. Marine Ecology Progressive Series, 388, 235-242.

Munday, P.L., McCormick, M.I. and Nilsson, G.E. (2012). Impact of global warming and rising $\mathrm{CO} 2$ levels on coral reef fishes: what hope for the future? Journal of Experimental Biology, 215 , 3865-3873.

Metcalfe, N.B., Huntingford, F.A., Thorpe, J.E. and Adams, C.E. (1990). The effects of social status on life-history variation in juvenile salmon. Canadian Journal of Zoology, 68, 2630-2636.

Michelangeli, M., Goulet, C. T., Kang, H. S., Wong, B. B. and Chapple, D. G. (2018). Integrating thermal physiology within a syndrome: locomotion, personality and habitat selection in an ectotherm. Functional Ecology, 32, 970-981.

Midwood, J.D., Larsen, M.H., Boel, M., Aarestrup, K. and Cooke, S.J. (2015). An experimental field evaluation of winter carryover effects in semi- anadromous brown trout (Salmo trutta).

Journal of Experimental Zoology Part A: Ecological and Integrative Physiology, 323, 645-654.

Morgan, R., Sundin, J., Finnøen, M.H., Dresler, G., Martinez Vendrell, M., Dey, A., Sarkar, K. and Jutfelt, F. (2019). Are model organisms representative for climate change research? Testing 
thermal tolerance in wild and laboratory zebrafish populations. Conservation Physiology, 7, 111 .

Morgan, R., Finnøen, M.H. and Jutfelt, F. (2018). CT $\mathrm{T}_{\max }$ is repeatable and doesn't reduce growth in zebrafish. Science Reports, 8, 7099.

Morinville, G.R. and Rasmussen, J.B. (2003). Early juvenile bioenergetics differences between anadromous and resident brook trout (Salvelinus alpinus) in Norway. Canadian Journal of Fisheries and Aquatatic Sciences, 40,1372-1387.

Muñoz, N.J., Farrell, A.P., Heath, J.W. and Neff, B.D. (2015). Adaptive potential of a Pacific salmon challenged by climate change. Nature Climate Change, 5,163-166.

Muñoz, M.M., Stimola, M.A., Algar, A.C., Conover, A., Rodriguez, A.J., Landestoy, M.A., Bakken, G.S. and Losos J.B. (2014). Evolutionary stasis and lability in thermal physiology in a group of tropical lizards. Proceedings of the Royal Society B, 281, 20132433.

Nielsen, C., Aarestrup, K. and Madsen, S.S. (2006). Comparison of physiological smolt status in descending and nondescending wild brown trout (Salmo trutta) in a Danish stream. Ecology of Freshwater Fishes, 15, 229-236.

Nielsen C., Aarestrup, K., Nørum, U. and Madsen, S.S. (2004). Future migratory behaviour predicted from premigratory levels of gill $\mathrm{Na}+/ \mathrm{K}+$-ATPase activity in individual wild brown trout (Salmo trutta). Journal of Experimental Biology, 207, 527-533.

Nilsson, A.L.K., Alerstam, T. and Nilsson, J-A. (2008). Diffuse, short and slow migration among Blue Tits. Journal of Ornithology, 149, 365-373.

Nilsson, G.E., Crawley, N., Lunde, I.G. and Munday, P.L. (2009). Elevated temperature reduces the respiratory scope of coral reef fishes. Global Change Biology, 15, 1405-1412.

Nussey, D.H., Wilson, A.J. and Brommer, J.E. (2007). The evolutionary ecology of individual phenotypic plasticity in wild populations. Journal of evolutionary biology, 20, 831-844.

Nivelle, R., Gennotte, V., Kalala, E.J.K., Ngoc, N.B., Muller, M., Mélard, C. and Rougeot, C. (2019). Temperature preference of Nile tilapia (Oreochromis niloticus) juveniles induces spontaneous sex reversal. PLoS One, 14, 1-19.

O'Donnell, M.J., Regish, A.M., McCormick, S.D. and Letcher, B.H. (2020). How repeatable is CTmax within an individual brook trout over short and long-time intervals? Journal of Thermal Biology 102559.

Økland, F., Jonsson, B., Jensen, A.J. and Hansen, L.P. (1993). Is there a threshold size regulating seaward migration of brown trout and Atlantic salmon? Journal of Fish Biology, 42, 541-550. 
Olsson, I.C., Greenberg, L.A., Bergman, E. and Wysujack, K. (2006). Environmentally induced migration: the importance of food. Ecology Letters, 9, 645-651.

Parmesan, C. and Yohe, G. (2003). A globally coherent fingerprint of climate change impacts across natural systems. Nature, 421, 37-42.

Payne, N.L., Morley, S.A., Halsey, L.G., Smith, J.A., Stuart-Smith, R., Waldock, C. and Bates, A.E. (2021). Fish heating tolerance scales similarly across individual physiology and populations. Communications biology, 4, pp.1-5.

Peck, L.S. (2011). Organisms and responses to environmental change. Marine genomics, 4, 237243.

Peiman, K.S., Birnie-Gauvin, K., Midwood, J.D., Larsen, M.H., Wilson, A.D.M., Aarestrup, K. and Cooke, S.J. (2017). If and when: intrinsic differences and environmental stressors influence migration in brown trout (Salmo trutta). Oecologia, 184, 375-384.

Penn, J.L., Deutsch, C., Payne, J.L. and Sperling, E.A. (2018). Temperature-dependent hypoxia explains biogeography and severity of end-Permian marine mass extinction. Science, 362, eaat1327.

Perkins, S.E., Alexander, L.V. and Nairn, J.R. (2012). Increasing frequency, intensity and duration of observed global heatwaves and warm spells. Geophysical Research Letters, 39. Perry, A.L., Low, P.J., Ellies, J.R. and Reynolds, J.D. (2005). Climate Change and Distribution Shifts in Marine Fishes. Science, 308, 1912-1915.

Perry, G.M., Martyniuk, C.M., Ferguson, M.M. and Danzmann, R.G. (2005). Genetic parameters for upper thermal tolerance and growth-related traits in rainbow trout (Oncorhynchus mykiss). Aquaculture, 250, 120-128.

Pinsky, M.L., Eikeset, A.M., McCauley, D.J., Payne, J.L. and Sunday, J.M. (2019). Greater vulnerability to warming of marine versus terrestrial ectotherms. Nature, 569, 108-111.

Pörtner, H.O. (2002). Physiological basis of temperature-dependent biogeography: trade-offs in muscle design and performance in polar ectotherms. Journal of Experimental Biology, 205, 2217-2230.

Pörtner, H.O. and Knust, R. (2007). Climate change affects marine fishes through the oxygen limitation of thermal tolerance. Science, 315, 95-97.

Pörtner, H.O., Bock, C., Knust, R., Lannig, G., Lucassen, M., Mark, F.C. and Sartoris, F.J. (2008). Cod and climate in a latitudinal cline: physiological analyses of climate effects in marine fishes. Climate Research, 37, 253-270.

Pörtner, H.O. and Farrell, A.P. (2008). Ecology. Physiology and climate change. Science, 322, 690-692. 
Pörtner, H.O. (2010). Oxygen- and capacity-limitation of thermal tolerance: a matrix for integrating climate-related stressor effects in marine ecosystems. Journal of Experimental Biology, 213, 881-893.

Pörtner, H.O. and Peck, M.A. (2010). Climate change effects on fishes and fisheries: towards a cause-and-effect understanding. Journal of Fish Biology, 77, 1745-1779.

Pörtner, H.O., Bock, C. and Mark, F.C. (2017). Oxygen-and capacity-limited thermal tolerance: bridging ecology and physiology. Journal of Experimental Biology, 220, 2685-2696.

Potts, L. (2020). Hot and Already Bothered: Exploring Effects of Warming Waters on an Imperiled Freshwater Fish, Pugnose Shiner «Notropis Anogenus» (Doctoral dissertation, McGill University Libraries).

R Core Team. (2016). R: A language and environment for statistical computing. R Foundation for Statistical Computing, Vienna, Austria.

Rezende, E.L., Tejedo, M. and Santos, M., (2011). Estimating the adaptive potential of critical thermal limits: methodological problems and evolutionary implications. Functional Ecology, 25, 111-121.

Rezende E.L., Castañeda L.E. and Santos M. (2014) Tolerance landscapes in thermal ecology. Functional Ecology, 28, 799-809.

Rezende, E.L. and Bozinovic, F. (2019). Thermal performance across levels of biological organization. Philosophical Transactions of the Royal Society B, 374, 20180549.

Recsetar, M.S., Zeigler, M.P., Ward, D.L., Bonar, S.A. and Caldwell, C.A. (2012). Relationship between fish size and upper thermal tolerance. Transactions of the American Fisheries Society, 141, 1433-1438.

Ricker, W.E. (1975). Computation and interpretation of biological statistics of populations. Bulletin of the Fisheries Research Board of Canada, 191, 382.

Rodgers, E.M., Todgham, A.E., Connon, R.E. and Fangue, N.A. (2019). Stressor interactions in freshwater habitats: Effects of cold water exposure and food limitation on early-life growth and upper thermal tolerance in white sturgeon, Acipenser transmontanus. Freshwater Biology, 64, 348-358.

Sandblom, E., Clark, T.D., Gräns, A., Ekström, A., Brijs, J., Sundström, L.F., Odelström, A., Adill, A., Aho, T. and Jutfelt, F. (2016). Physiological constraints to climate warming in fish follow principles of plastic floors and concrete ceilings. Nature Communications, 7, 1-8. 
Sardella, B.A., Sanmarti, E. and Kültz, D. (2008). The acute temperature tolerance of green sturgeon (Acipenser medirostris) and the effect of environmental salinity. Journal of Experimental Zoology Part A: Ecological Genetics and Physiology, 309, 477-483.

Sarkar, D. (2008). Lattice: Multivariate Data Visualization with R. Springer, New York. ISBN 978-0-387-75968-5.

Schulte, P.M., Healy, T.M. and Fangue, N.A. (2011). Thermal performance curves, phenotypic plasticity, and the time scales of temperature exposure. Integrative and comparative biology, 51, 691-702.

Seneviratne, S.I., Donat, M.G., Mueller, B. and Alexander, L.V. (2014). No pause in the increase of hot temperature extremes. Nature Climate Change, 4(3), pp.161-163.

Sinclair, B.J., Marshall, K.E., Sewell, M.A., Levesque, D.L., Willett, C.S., Slotsbo, S., Dong, Y., Harley, C.D., Marshall, D.J., Helmuth, B.S. and Huey, R.B. (2016). Can we predict ectotherm responses to climate change using thermal performance curves and body temperatures? Ecology Letters, 19, 1372-1385.

Sloat, M.R. and Reeves, G.H. (2014). Individual condition, standard metabolic rate, and rearing temperature influence steelhead and rainbow trout (Oncorhynchus mykiss) life histories. Canadian Journal of Fisheries and Aquatic Sciences, 71, 491-501.

Somero, G.N. 2010. The physiology of climate change: how potentials for acclimatization and genetic adaptation will determine 'winners' and 'losers'. Journal of Experimental Biology, 213, 912-920.

Steinhausen, M.F., Sandblom, E., Eliason, E.J., Verhille, C. and Farrell, A.P. (2008). The effect of acute temperature increases on the cardiorespiratory performance of resting and swimming sockeye salmon (Oncorhynchus nerka). Journal of Experimental Biology, 211, 3915-3926.

Stevens, E.D. and Fry, F.E.J. (1974). Heat transfer and body temperatures in nonthermoregulatory teleosts. Canadian journal of zoology, 52, 1137-1143.

Sunday, J.M., Bates, A.E. and Dulvy, N.K. (2012). Thermal tolerance and the global redistribution of animals. Nature Climate Change, 2, 686-690.

Sunday, J.M., Bates, A.E., Kearney, M.R., Colwell, R.K., Dulvy, N.K., Longino, J.T. and Huey, R.B. (2014). Thermal-safety margins and the necessity of thermoregulatory behavior across latitude and elevation. Proceedings of the National Academy of Sciences, 111, 5610-5615.

Sunday, J.M., Bennett, J.M., Calosi, P., Clusella-Trullas, S., Gravel, S., Hargreaves, A.L., Leiva, F.P., Verberk, W.C.E.P., Olalla-Tárraga, M.Á. and Morales-Castilla, I. (2019). Philosophical Transactions of the Royal Society B, 374, 20190036. 
Terblanche, J.S., Deere, J.A., Clusella-Trullas, S., Janion, C. and Chown, S.L. (2007). Critical thermal limits depend on methodological context. Proceedings of the Royal Society B: Biological Sciences, 274, 2935-2943.

Theriault, V. and Dodson, J.J. (2003). Body size and the adoption of a migratory tactic in brook charr. Journal of Fish Biology, 63, 1144-1159.

Thomas, C.D., Bodsworth, E.J., Wilson, R.J., Simmons, A.D., Davies, Z.G., Musche, M. and Conradt. L. (2001). Ecological and evolutionary processes at expanding range margins. Nature, $411,577-581$.

Thorpe, J.E., Mangel, M., Metcalfe, N.B. and Huntingford, F.A. (1998). Modelling the proximate basis of salmonid life-history variation, with application to Atlantic salmon, Salmo salar L. Evolutionary Ecology, 12, 581-599.

Turko, A.J., Nolan, C.B., Balshine, S., Scott, G.R. and Pitcher, T.E. (2020). Thermal tolerance depends on season, age and body condition in imperilled redside dace Clinostomus elongatus. Conservation Physiology, 8(1), p.coaa062.

Venables, W.N. and Ripley, B.D. (2002). Modern Applied Statistics with S. Fourth Edition. Springer, New York. ISBN 0-387-95457-0.

Vertessy, R., Barma, D., Baumgartner, L., Bond, N., Mitrovic, S. and Sheldon, F. (2019). Independent assessment of the 2018-19 fish deaths in the lower Darling.

Vinagre, C., Leal, I., Mendonça, V. and Flores, A.A. (2015). Effect of warming rate on the critical thermal maxima of crabs, shrimp and fish. Journal of thermal biology, 47, 19-25.

Vinagre, C., Dias, M., Cereja, R., Abreu-Afonso, F., Flores, A.A. and Mendonça, V. (2019). Upper thermal limits and warming safety margins of coastal marine species-Indicator baseline for future reference. Ecological Indicators, 102, 644-649.

Wang, G. and Dillon, M.E. (2014) Recent geographic convergence in diurnal and annual temperature cycling flattens global thermal profiles. Nature Climate Change, 4, 988-992.

Waldock, C., Dornelas, M. and Bates, A.E. (2018). Temperature-driven biodiversity change: disentangling space and time. Bioscience, 68, 873-884.

Whitney, J.E., Al-Chokhachy, R., Bunnell, D.B., Caldwell, C.A., Cooke, S.J., Eliason, E.J., Rogers, M., Lynch, A.J. and Paukert, C.P. 2016. Physiological basis of climate change impacts on North American inland fishes. Fisheries, 41, 332-345.

Wickham, H. 2016. ggplot2: Elegant Graphics for Data Analysis. Springer-Verlag New York. ISBN 978-3-319-24277-4. 
Williams, C.M., Buckley, L.B., Sheldon, K.S., Vickers, M., Pörtner, H.O., Dowd, W.W., Gunderson, A.R., Marshall, K.E. and Stillman, J.H. (2016). Biological impacts of thermal extremes: mechanisms and costs of functional responses matter. Integrative and comparative biology, 56, 73-84.

Wysujack, K., Greenberg, L. A., Bergman, E. and Olsson, I.C. (2009). The role of the environment in partial migration: food availability affects the adoption of a migratory tactic in brown trout Salmo trutta. Ecology of Freshwater Fishes, 18, 52-49.

Zhang, Y. and Kieffer, J.D. (2014). Critical thermal maximum (CTmax) and hematology of shortnose sturgeons (Acipenser brevirostrum) acclimated to three temperatures. Canadian Journal of Zoology, 92, 215-221.

Zydlewski, G.B., Horton, G., Dubreuil, T., Letcher, B., Casey, S. and Zydlewski, J. (2006). Remote monitoring of fish in small streams: a unified approach using PIT tags. Fisheries, 31, 492-502. 